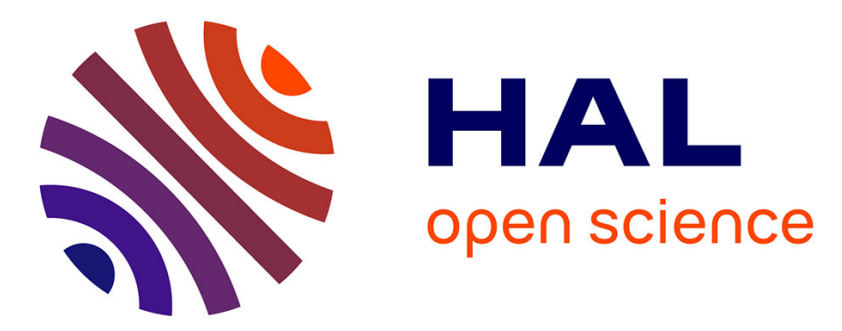

\title{
Wavelet analysis and covariance structure of some classes of non-stationary processes \\ Charles-Antoine Guérin
}

\section{To cite this version:}

Charles-Antoine Guérin. Wavelet analysis and covariance structure of some classes of nonstationary processes. Journal of Fourier Analysis and Applications, 2000, 6 (4), pp.403-425. 10.1007/BF02510146 . hal-00083193

\section{HAL Id: hal-00083193 https://hal.science/hal-00083193}

Submitted on $10 \mathrm{Jul} 2016$

HAL is a multi-disciplinary open access archive for the deposit and dissemination of scientific research documents, whether they are published or not. The documents may come from teaching and research institutions in France or abroad, or from public or private research centers.
L'archive ouverte pluridisciplinaire HAL, est destinée au dépôt et à la diffusion de documents scientifiques de niveau recherche, publiés ou non, émanant des établissements d'enseignement et de recherche français ou étrangers, des laboratoires publics ou privés. 


\title{
Wavelet Analysis and Covariance Structure of some Classes of Non-Stationary Processes
}

\author{
Charles-Antoine Guérin
}

\author{
Communicated by C. Houdré
}

\begin{abstract}
Processes with stationary n-increments are known to be characterized by the stationarity of their continuous wavelet coefficients. We extend this result to the case of processes with stationary fractional increments and locally stationary processes. Then we give two applications of these properties. First, we derive the explicit covariance structure of processes with stationary $n$-increments. Second, for fractional Brownian motion, the stationarity of the fractional increments of order greater than the Hurst exponent is recovered.
\end{abstract}

\section{Introduction}

The aim of this paper is to present a unified approach to the wavelet analysis of some classes of non-stationary stochastic processes: processes with stationary $n$-increments, processes with stationary fractional increments, and locally stationary processes. We will give a characterization of each class in terms of continuous wavelet transform and then use this to derive some non-trivial second-order properties of these processes.

The wavelet analysis of stochastic processes has been developed in the last decade, mainly in connection with self-similar processes like fractional Brownian motion: e.g., [10], [35], [24] for the continuous wavelet analysis, and [1], [40], [7], [8], [11], [25], [39], [42], [6], [20], [2] for the discrete wavelet transform. Recently, many correspondences have been established between the second-order [19], [5] and distributional properties [3] of a process and its continuous wavelet transform. In particular, a convenient characterization of processes with stationary $n$ th-increments has been given: a process has stationary $n$ th-increments if and only if its wavelet transform is stationary for a wavelet with $n$ vanishing moments. We will extend these results to the processes with stationary fractional increments and locally stationary processes.

The results of the present work can be summarized in the following way. A process has stationary fractional increments if and only if its wavelet transform is stationary (Theorem 4); a

Math Subject Classifications. 42A38, 42A82, 46F, 46N30, 60G12.

Keywords and Phases. wavelet analysis, stationary processes, locally stationary processes, stationary increments, fractional increments, fractional Brownian motion.

Acknowledgements and Notes. The author has been supported by a grant of Chalmers University, Gothenborg, Sweden. 
process is locally stationary if and only if its wavelet transform is locally stationary (Theorem 6), where the analyzing wavelet has to be adapted to every case. As an application, we derive the explicit covariance structure of stationary $n$th increments (Theorem 3) and show that fractional Brownian motion with Hurst parameter $H$ has stationary $\alpha$-increments for $\alpha>H$ (Theorem 5). We have chosen to detail all the proofs which follow a logical order in the body of the paper and relegate all technical independent results to the appendices.

Before entering the core of the paper, we give a brief overview of the different classes of processes we want to consider.

Processes with Stationary $n$-Increments. Processes with stationary $n$-increments are perhaps the simplest class of processes with non-trivial non-stationarity and are often used for modelization in physics. Let $X(t)$ be a complex-valued random process on $\mathbb{R}$. For any $\tau \in \mathbb{R}$, we define the shift operator $B_{\tau}$ by

$$
B_{\tau} X(t)=X(t-\tau)
$$

and the discrete differentiation operator $\Delta_{\tau}$ by $\Delta_{\tau}=1-B_{\tau}$. The $n$th difference operator $\Delta_{\tau}^{n}$ can be simply expressed by means of the Binomial coefficients

$$
\Delta_{\tau}^{n}=\left(1-B_{\tau}\right)^{n}=\sum_{j=0}^{n}(-1)^{j}\left(\begin{array}{c}
n \\
j
\end{array}\right) B_{\tau}^{j} .
$$

A process $X(t)$ is said to have stationary $n$th increments if for all $s, t, \tau, \tau^{\prime}$ the expectations $\mathbb{E} \Delta_{\tau}^{n} X(s)$ and $\mathbb{E} \Delta_{\tau}^{n} X(s) \overline{\Delta_{\tau^{\prime}}^{n} X(s+t)}$ exist ${ }^{1}$ and do not depend on $s$. As can be easily checked, this amounts to the following definition.

Definition 1. A process $X$ is said to have stationary $n$th increments if $\Delta_{\tau}^{n} X$ is (wide sense) stationary for all $\tau \in \mathbb{R}$.

The main results for this class of processes were derived in the 1950s: Yaglom and Pinsker [43], [45], [44], [34], [33] established a spectral representation based on Khinchine theorem, while Gel'fand [15], [13] obtained an analogous result in the framework of generalized random processes, using the spectral representation of conditionally positive definite generalized functions. Since then, the only extensions we are aware of are re-formulations of the spectral representation in the framework of linear filtering theory [32] and semi-martingale representation [41]. A process with stationary $n$th increments can be written as

$$
X(t)=\int\left(e^{i \xi t}-1-i \xi t \cdots-\frac{(i \xi t)^{n-1}}{(n-1) !}\right) d Y(\xi)+\Upsilon_{0}+\Upsilon_{1} t+\ldots \Upsilon_{n} t^{n},
$$

where the $\Upsilon_{j}$ are random variables and $Y$ is a process with orthogonal increments such that

$$
\int \frac{\xi^{2 n}}{1+\xi^{2 n}} \mathbb{E}|d Y(\xi)|^{2}<\infty
$$

To find the correlation $\mathbb{E} X(s) \overline{X(t)}$ we need to compute the integral

$$
\int\left(e^{i \xi s}-1-i \xi s \cdots-\frac{(i \xi s)^{n-1}}{(n-1) !}\right)\left(e^{i \xi t}-1-i \xi t \cdots-\frac{(i \xi t)^{n-1}}{(n-1) !}\right) \mathbb{E}|d Y(\xi)|^{2}
$$

\footnotetext{
${ }^{1}$ The symbol $\bar{X}$ stands for the complex conjugation.
} 
It is a convergent sum of divergent integrals, which makes it difficult to develop and reorder into different integrals. Furthermore, the interdependence of the $\Upsilon_{j}$ and the spectral measure is not known. Therefore, it seems to us that the spectral representation is not very satisfactory, since it does not reveal the explicit structure of the covariance function and hence poorly describes the nonstationarity. To circumvent the difficulty, we propose use of the wavelet characterization of such processes. Roughly speaking, the processes with stationary $n$-increments can have a polynomially diverging spectral density at zero [see formula (1.3)]. The wavelet analysis is a good tool to study such processes, since the wavelet transform removes the low-frequency content of a signal. This idea was used in [10] for a rigorous definition of a spectrum of fractional Brownian motion.

Processes with Stationary Fractional Increments. Fractionally differentiated processes were introduced in [16] and [17] to construct the class of fractional ARIMA processes, which are a generalization of the classical ARIMA model with a fractional degree of differentiation and can exhibit long range correlations. Following [4], we define for any real number $-1<\alpha \notin \mathbb{N}$ the fractional difference operator $\Delta_{\tau}^{\alpha}$ by

$$
\Delta_{\tau}^{\alpha}=\left(1-B_{\tau}\right)^{\alpha}=\sum_{j=0}^{\infty} \pi_{j}^{\alpha} B_{\tau}^{j},
$$

where

$$
\pi_{0}^{\alpha}=1, \pi_{j}^{\alpha}=\frac{\Gamma(j-\alpha)}{\Gamma(j+1) \Gamma(-\alpha)}=\prod_{0<k \leq j} \frac{k-1-\alpha}{k}, j=1,2 \ldots,
$$

and $\Gamma$ is the Gamma function:

$$
\Gamma(x)= \begin{cases}\int_{0}^{\infty} t^{x-1} e^{-t} d t, & x>0 \\ \infty, & x=0 \\ x^{-1} \Gamma(1+x), & x<0\end{cases}
$$

The $\alpha$-increments of a process $X(t)$ are defined by $\Delta_{\tau}^{\alpha} X(t)$, whenever this series makes sense. To motivate the study of the fractional increments, it is important to replace them in the context of times series. We can make time series out of $X(t)$ and $\Delta_{\tau}^{\alpha} X$ by considering $X_{n}=X(t=n)$ and $\Delta^{\alpha} X_{n}=\left(\Delta_{\tau=1}^{\alpha} X\right)(t=n)$. A time series $X_{n}$ is said to be ARIMA $(0, \alpha, 0)$ if

$$
\Delta^{\alpha} X_{n}=Z_{n},
$$

where $Z_{n}$ is a white noise. Given $Z_{n}$ and $|\alpha|<0.5$, it is known [4] that there exists a unique stationary solution $X_{n}$ to (1.5). A natural question might be whether there exists non-stationary solutions. This raises the problem of determining those non-stationary processes, discrete or continuous, which become stationary after fractional differentiation. This yields to the following definition.

Definition 2. A process $X$ has stationary $\alpha$-increments if $\Delta_{\tau}^{\alpha} X$ is (wide sense) stationary for all $\tau \in \mathbb{R}$.

Again the wavelet analysis will turn out to be a very efficient tool to characterize such processes.

Locally Stationary Processes. Locally stationary processes were introduced by Silverman in [38] to describe physical systems whose statistical characteristics change slowly in time. A process is (wide sense) locally stationary if its covariance $C(s, t)$ can be written in the form

$$
C(s, t)=m\left(\frac{s+t}{\sqrt{2}}\right) \gamma\left(\frac{s-t}{\sqrt{2}}\right),
$$


where $\gamma$ is a stationary covariance function (the factor $1 / \sqrt{2}$ is not in the original definition but we put it in for convenience, see Section 5). Note that the condition $C(s, s) \geq 0$ forces the function $m$ to be non-negative. Furthermore, we can always assume that the covariance term is normalized, i.e., $\gamma(0)=1$. Locally stationary processes are very convenient to model non-stationarity because many properties of stationary processes can be translated to them [28], [26], [27]. This is why they can be successively characterized with the help of the wavelet transform by adapting the method developed for stationary processes. Note that recently a different and less restrictive notion of local stationarity has been introduced by means of orthogonal wavelets (see, e.g., [22] and references therein). However, we will restrict ourselves to the definition of Silverman, which is more tractable in the context of continuous wavelet analysis.

\section{Hypothesis and Definitions}

Hypothesis on the Processes. We consider a complex-valued measurable random process $(X(t)$, $t \in \mathbb{R})$ on some probability space $(\Omega, \mathcal{F}, P)$. The dependence on $\omega \in \Omega$ will always be implicit and the stationarity throughout will be understood in the wide sense. Further, the process will be mean-square continuous (m.s continuous), which, as it is well known, is equivalent to assuming the continuity of the covariance function in its two variables. We will denote by $M(t)$ and $C(s, t)$ the mean and covariance of the process, respectively,

$$
\begin{aligned}
M(t) & =\mathbb{E} X(t), \\
C(s, t) & =\mathbb{E} X(s) \overline{X(t)}-\mathbb{E} X(s) \mathbb{E} \overline{X(t)}
\end{aligned}
$$

To be able to define the wavelet transform, we will assume the following growth restriction on the second moment function:

$$
\mathbf{H}:|\mathbb{E} X(s) \overline{X(t)}| \leq O\left(\left(1+|s|^{m}\right)\left(1+|t|^{m}\right)\right) \text { for some } m>0
$$

This implies in particular that the variance and the mean are polynomially bounded. Note that this condition is automatically satisfied if the process has $n$th stationary increments, as can easily be shown.

Some Functional Spaces. Following the usual convention, we denote $\mathcal{D}\left(\mathbb{R}^{n}\right)$ the space of complexvalued $C^{\infty}$ functions on $\mathbb{R}^{n}$ with compact support and $\mathcal{S}\left(\mathbb{R}^{n}\right)$ the space of Schwartz functions on $\mathbb{R}^{n}$, that is the $C^{\infty}$ functions $\phi$ which are, together with their derivatives, rapidly decreasing at infinity:

$$
\sup _{\left(x_{1}, \ldots x_{n}\right) \in \mathbb{R}^{n}}\left|x_{1}\right|^{\alpha_{1}} \ldots\left|x_{n}\right|^{\alpha_{n}}\left|D_{1}^{\beta_{1}} \ldots D_{n}^{\beta_{n}} \phi\left(x_{1}, \ldots x_{n}\right)\right|<\infty \text { for all } \alpha_{j}, \beta_{j} \in \mathbb{N} .
$$

We will use the notation $D$ for the distributional derivative on $\mathbb{R}$ and $D_{s}$ and $D_{t}$ for the partial distributional derivatives on $\mathbb{R}^{2}$. The dual $\mathcal{D}^{\prime}\left(\mathbb{R}^{n}\right)$ is the space of generalized functions and $\mathcal{S}^{\prime}\left(\mathbb{R}^{n}\right)$ the space of tempered distributions on $\mathbb{R}^{n}$. Under our assumptions, the covariance function $C(s, t)$ can be considered as a distribution in $\mathcal{S}^{\prime}\left(\mathbb{R}^{2}\right)$. We use the notation $\widehat{\phi}$ for the Fourier transform of a function $\phi$ in $\mathcal{S}\left(\mathbb{R}^{n}\right)$,

$$
\widehat{\phi}\left(\xi_{1}, \ldots, \xi_{n}\right)=(2 \pi)^{-n / 2} \int e^{-i\left(\xi_{1} x_{1}+. . \xi_{n} x_{n}\right)} \phi\left(x_{1}, \ldots, x_{n}\right) d x_{1} \ldots d x_{n},
$$

and the same notation for the Fourier transform of tempered distributions, $\widehat{\mu}(\phi)=\mu(\widehat{\phi})$, the distinction being clear from the context. The term "measure" will always refer to Borel measures on $\mathbb{R}^{n}$ assuming finite mass on compact sets, which can be identified with distributions of order 0 in $\mathcal{D}^{\prime}\left(\mathbb{R}^{n}\right)$. 
If a measure $\mu$ is of tempered growth, i.e., $\int\left(1+\|x\|^{l}\right)^{-1} d|\mu|<\infty$ for some $l>0$, then it can also be identified with a distribution of order 0 in $\mathcal{S}^{\prime}(\mathbb{R})$, that is such that $|\mu(\phi)| \leq K\left\|\left(1+\|x\|^{l}\right) \phi(x)\right\|_{\infty}$ for all $\phi \in \mathcal{S}(\mathbb{R})$. In the following, we will frequently make use of approximate identities, whose definition we recall hereafter.

Definition 3. An approximate identity in $\mathcal{S}^{\prime}\left(\mathbb{R}^{n}\right)$ is a family of functions $\phi_{a}(x)=a^{-1} \phi\left(a^{-1} x\right)$ with $\phi \in \mathcal{S}\left(\mathbb{R}^{n}\right)$ and $\int \phi=1$. If in addition $\phi \in \mathcal{D}\left(\mathbb{R}^{n}\right)$ and $\phi \geq 0$, then $\phi_{a}$ is an approximate identity in $\mathcal{D}^{\prime}\left(\mathbb{R}^{n}\right)$.

If $\phi_{a}$ is an approximate identity in $\mathcal{S}^{\prime}\left(\mathbb{R}^{n}\right)$, it is well known (see, e.g., [12]) that for any $\psi \in \mathcal{S}\left(\mathbb{R}^{n}\right)$ and $F \in \mathcal{S}^{\prime}\left(\mathbb{R}^{n}\right), \phi_{a} * \psi \rightarrow \psi$ in $\mathcal{S}\left(\mathbb{R}^{n}\right)$, and $\phi_{a} * F \rightarrow F$ in $\mathcal{S}^{\prime}\left(\mathbb{R}^{n}\right)$. If $\phi_{a}$ is an approximate identity in $\mathcal{D}^{\prime}\left(\mathbb{R}^{n}\right)$, the analogous statement holds in the topologies of $\mathcal{D}\left(\mathbb{R}^{n}\right)$ and $\mathcal{D}^{\prime}\left(\mathbb{R}^{n}\right)$. If $\psi$ is only a continuous tempered function and $\phi$ is either in $\mathcal{S}\left(\mathbb{R}^{n}\right)$ or $\mathcal{D}\left(\mathbb{R}^{n}\right)$, then we have $\phi_{a} * \psi \rightarrow \psi$ for the pointwise convergence.

Wavelet Transform of Random Processes. For all $(b, a) \in \mathbb{R}^{n} \times \mathbb{R}^{+}$, we will adopt the following notation for the symmetric, resp. dilated, resp. dilated and translated version, of a function $g$ on $\mathbb{R}^{n}$ :

$$
\tilde{g}(x)=\overline{g(-x)}, g_{a}(x)=\frac{1}{a^{n}} g\left(\frac{x}{a}\right), g_{b, a}(x)=\frac{1}{a^{n}} g\left(\frac{x-b}{a}\right) .
$$

For all $g \in \mathcal{S}(\mathbb{R})$ and $(b, a) \in \mathbb{R} \times \mathbb{R}^{+}$, the process $\bar{g}_{b, a}(t) X(t)$ is measurable with

$$
\int \mathbb{E}\left|\bar{g}_{b, a}(t) X(t)\right| d t<\infty \text {. }
$$

It follows (see, e.g., [9]) that almost surely the sample functions of $\bar{g}_{b, a}(t) X(t)$ are Lebesgue integrable and we may define the integral:

$$
\mathcal{W}_{g} X(b, a)=\int \frac{1}{a} \bar{g}\left(\frac{t-b}{a}\right) X(t) d t=\left(\tilde{g}_{a} * X\right)(b),
$$

which we call the wavelet transform of $X$ with respect to the wavelet $g$ (even if $g$ is not truly a wavelet in the sense that $\int g=0$ ). For fixed $a$, the wavelet transform defines a random process $\mathcal{W}_{g} X(\cdot, a)$ on $\mathbb{R}$, which we refer to as the "wavelet process." Its mean $M_{a}$ and covariance $C_{a}(s, t)$ are given by

$$
\begin{aligned}
M_{a}(t) & =\mathbb{E} \mathcal{W}_{g} X(t, a) \\
& =\int \frac{1}{a} \bar{g}\left(\frac{t^{\prime}-t}{a}\right) M\left(t^{\prime}\right) d t^{\prime} \\
& =\tilde{g}_{a} * M(t), \\
C_{a}(s, t) & =\mathbb{E} \mathcal{W}_{g} X(s, a) \overline{\mathcal{W}_{g} X(t, a)}-\mathbb{E} \mathcal{W}_{g} X(s, a) \mathbb{E} \overline{\mathcal{W}_{g} X(t, a)} \\
& =\int \frac{1}{a} \bar{g}\left(\frac{s^{\prime}-s}{a}\right) \frac{1}{a} g\left(\frac{t^{\prime}-t}{a}\right) C\left(s^{\prime}, t^{\prime}\right) d s^{\prime} d t^{\prime} \\
& =\left(\widetilde{G}_{a} * C\right)(s, t),
\end{aligned}
$$

where the last convolution acts in the plane and $G(s, t)=g(s) \bar{g}(t)$. The exchange of expectation and integration in both formula is justified by Fubini's theorem, since the integrals are absolutely convergent. Thus, mean and covariance of the process in the wavelet plane are merely smooth versions of the original mean and covariance. They can also be seen as the one- and two-dimensional wavelet transforms of these latter with respect to the wavelet $g$ and $G$. We will often consider Equations (2.1) and (2.2) on the Fourier side; that is,

$$
\begin{aligned}
\widehat{M_{a}}(\xi) & =\widehat{\widehat{g}}(a \xi) \widehat{M}(\xi) \\
\widehat{C_{a}}(\xi, \eta) & =\widehat{\vec{g}}(a \xi) \widehat{g}(-a \eta) \widehat{C}(\xi, \eta)
\end{aligned}
$$


Some Classes of Wavelets. The wavelet to be chosen for the wavelet analysis will depend on the process under consideration. Let us introduce the following classes of wavelets.

Wavelets with no Vanishing Moments. We say that a "wavelet" $g \in \mathcal{S}(\mathbb{R})$ is in the class $\mathcal{S}_{0}(\mathbb{R})$ if $\int g \neq 0$. We say that $g$ is a Gaussian "wavelet" if $g(x)=\alpha e^{-\beta x^{2}}$ for some complex numbers $\alpha$ and $\beta$ with $\Re \beta>0$, where $\Re z$ denotes the real part of $\mathrm{c}$ a complex number $z$.

Wavelets with $n$ Vanishing Moments. For any integer $n \geq 1$, we say that a wavelet $g \in \mathcal{S}(\mathbb{R})$ is in the class $\mathcal{S}_{\geq n}(\mathbb{R})$ if it has at least $n$ vanishing moments or, what amounts to the same, if its Fourier transform has a zero of order at least $n$ at the origin:

$$
\int x^{m} g(x) d x=0, m=0,1, \ldots, n-1 \Leftrightarrow|\widehat{g}(\xi)| \leq O\left(\xi^{n}\right), \xi \rightarrow 0 .
$$

We say that a wavelet $g \in \mathcal{S}(\mathbb{R})$ is in the class $\mathcal{S}_{=n}(\mathbb{R})$ if it has exactly $n$ vanishing moments or, what amounts to the same, if its Fourier transform has a zero of order $n$ at the origin, ${ }^{2}$

$$
\int x^{m} g(x) d x=0, m=0,1, \ldots, n-1, \text { and } \int x^{n} g(x) d x \neq 0 \Leftrightarrow \widehat{g}(\xi) \sim \xi^{n}, \xi \rightarrow 0 \text {. }
$$

Wavelets with Fractional Number of Vanishing Moments. For any real $\alpha \geq 0$, we say that a wavelet $g$ is in the "fractional moment" space $\mathcal{F}_{\geq \alpha}(\mathbb{R})$ if $\widehat{g}$ can be written as

$$
\widehat{g}(\xi)=e^{i \frac{\alpha \pi}{2} \operatorname{sign}(\xi)}|\xi|^{\alpha} \widehat{\chi}(\xi)
$$

for some function $\chi \in \mathcal{S}(\mathbb{R})$. We say $g$ is in $\mathcal{F}_{=\alpha}(\mathbb{R})$ if $\chi \in \mathcal{S}_{0}(\mathbb{R})$.

As we will see, wavelets in $\mathcal{S}_{0}(\mathbb{R}), \mathcal{S}_{=n}(\mathbb{R})$, and $\mathcal{F}_{=\alpha}(\mathbb{R})$ will, respectively, characterize stationary processes, processes with stationary $n$th increments, and processes with stationary fractional increments. The Gaussian wavelets will characterize locally stationary processes.

Note that $\mathcal{F}_{\geq \alpha}(\mathbb{R})$ and $\mathcal{F}_{=\alpha}(\mathbb{R})$ reduce to the spaces $\mathcal{S}_{\geq n}(\mathbb{R})$ and $\mathcal{S}_{=n}(\mathbb{R})$ when $\alpha=n$ is an integer. The reason for this intricate definition will become clear in Section 4. The wavelets in $\mathcal{F}_{\geq \alpha}(\mathbb{R})$ and $\mathcal{F}_{=\alpha}(\mathbb{R})$ are not in the Schwartz space when $\alpha$ is non-integer. However, they have all the needed properties for our purposes.

\section{Processes with Stationary $\boldsymbol{n}$-Increments}

Stationary processes constitute the reference processes for all the following and therefore it is natural to start with them. It is clear from (2.1) and (2.2) that the wavelet process is stationary as soon as the original process is itself stationary. The converse is also true, as was shown in [5].

\section{Theorem 1.}

Let $X$ be an m.s continuous process on $\mathbb{R}$ satisfying $\mathbf{H}$.

i) If $X$ is stationary, then for all $g \in \mathcal{S}(\mathbb{R}), \mathcal{W}_{g} X(\cdot, a)$ is stationary for all $a>0$.

ii) Conversely, if for some $g \in \mathcal{S}_{0}(\mathbb{R}), \mathcal{W}_{g} X(\cdot, a)$ is stationary for all $a>0$, then $X$ is stationary.

iii) Under the stronger assumption $\widehat{g}(\xi) \neq 0$ for all $\xi$, it is enough that $\mathcal{W}_{g} X(\cdot, a)$ be stationary for some arbitrary $a>0$, for $X$ to be stationary.

\footnotetext{
${ }^{2}$ Here and everywhere, the symbol $\sim$ refers to the equivalence of two functions: $f \sim g$ if $c_{1} \leq f / g \leq c_{2}$ for some constants $0<c_{1} \leq c_{2}$.
} 
A similar result holds for processes with stationary $n$-increments, provided the wavelet has $n$ vanishing moments. This is not surprising, since such a wavelet performs a smooth differentiation of the process.

\section{Theorem 2.}

Let $X$ be an m.s continuous process on $\mathbb{R}$ satisfying $H$.

i) If $X$ has stationary $n$th increments, then the wavelet process $\mathcal{W}_{g} X(\cdot, a)$ is stationary for all wavelet $g$ in $\mathcal{S}_{\geq n}(\mathbb{R})$ and for all $a>0$.

ii) Conversely, if for some wavelet $g$ in $\mathcal{S}_{=n}(\mathbb{R}), \mathcal{W}_{g} X(\cdot, a)$ is stationary for all a $>0$, then $X$ has stationary nth increments.

iii) Under the stronger assumption $\widehat{g}(\xi) \neq 0$ for all $\xi \neq 0$, it is enough that $\mathcal{W}_{g} X(\cdot, a)$ be stationary for some arbitrary $a>0$, for $X$ to have stationary nth increments.

Proof. Also [5].

We will now use the above wavelet characterizations to derive the explicit covariance structure of processes with stationary $n$th increments. We start by the following lemma.

\section{Lemma 1.}

If $X$ is an m.s continuous process with stationary $n$th increments, then its covariance satisfies

$$
(-1)^{n} \xi^{n} \eta^{n} \widehat{C}(\xi, \eta)=d \mu(\xi) \delta(\xi+\eta)
$$

for some positive tempered measure $\mu$ such that

$$
\int\left(1+\xi^{2 n}\right)^{-1} d \mu(\xi)<\infty
$$

Here and everywhere, $\delta(\xi+\eta) d \mu(\xi)$ is the symbolic notation for the measure $m$ on $\mathbb{R}^{2}$ given by $m(\Phi)=\int d \mu(\xi) \Phi(\xi,-\xi)$.

Proof. Denote $\widehat{g}(\xi)=\xi^{n} e^{-\xi^{2}}$. Since $g$ is in $\mathcal{S}_{=n}(\mathbb{R})$, Theorem 2 implies that the wavelet process $\mathcal{W}_{g} X(\cdot, a)$ is stationary and thus its covariance satisfies

$$
\widehat{C_{a}}(\xi, \eta)=\delta(\xi+\eta) d \mu_{a}(\xi),
$$

for some finite positive measure $d \mu_{a}$. It follows from (2.4) that

$$
\begin{aligned}
(-1)^{n} \xi^{n} \eta^{n} \widehat{C}(\xi, \eta) & =(-1)^{n} a^{-2 n} \widehat{C_{a}}(\xi, \eta) e^{a^{2} \xi^{2}} e^{a^{2} \eta^{2}} \\
& =a^{-2 n} e^{2 a^{2} \xi^{2}} \delta(\xi+\eta) d \mu_{a}(\xi) .
\end{aligned}
$$

The above equality holds a priori in $\mathcal{D}^{\prime}\left(\mathbb{R}^{2}\right)$. However, the left-hand side is a tempered distribution, and does not depend on $a$. Thus, the right-hand side is also a tempered distribution in $\mathcal{S}^{\prime}\left(\mathbb{R}^{2}\right)$, independent of $a$ :

$$
(-1)^{n} \xi^{n} \eta^{n} \widehat{C}(\xi, \eta)=e^{2 \xi^{2}} \delta(\xi+\eta) d \mu_{1}(\xi)=: d \mu(\xi) \delta(\xi+\eta),
$$

where, by Lemma $5 \mathrm{i}$ ), $\mu$ is a tempered measure on $\mathbb{R}$. It remains to prove (3.2). For this, it suffices to show that $\int \xi^{-2 n}[1-\chi(\xi) \chi(-\xi)] d \mu(\xi)<\infty$, where $\chi$ is a cut-off function in $C_{0}^{\infty}(\mathbb{R})$ such that $\chi(\xi)=1$ for $|\xi| \leq 1$ and $0 \leq \chi \leq 1$. Now let $\Phi(s, t)=2^{-1} \exp \left(-\left(s^{2}+t^{2}\right) / 4\right)$. Since $\widehat{\Phi}(\xi, \eta)=\exp \left(-\xi^{2}-\eta^{2}\right)$ we have

$$
\int \xi^{-2 n}[1-\chi(\xi) \chi(-\xi)] d \mu(\xi)=\lim _{a \rightarrow 0} \int \widehat{\Phi_{a}}(\xi,-\xi) \xi^{-2 n}[1-\chi(\xi) \chi(-\xi)] d \mu(\xi),
$$


the above limit being finite or not. On the other hand, $\Phi_{a}$ is an approximate identity in $\mathcal{S}^{\prime}\left(\mathbb{R}^{2}\right)$, and thus by (3.4),

$$
\begin{aligned}
\lim _{a \rightarrow 0} \int \xi^{-2 n}[1-\chi(\xi) \chi(-\xi)] \widehat{\Phi_{a}}(\xi,-\xi) d \mu(\xi) & =\lim _{a \rightarrow 0} \widehat{C}\left(\widehat{\Phi_{a}}(1-\chi \otimes \chi)\right) \\
& =\lim _{a \rightarrow 0} \widehat{C}\left(\widehat{\Phi_{a}}\right)-\widehat{C}\left(\widehat{\Phi_{a}} \chi \otimes \chi\right) \\
& =\lim _{a \rightarrow 0} C\left(\Phi_{a}\right)-C\left(\Phi_{a} *(\widehat{\chi} \otimes \widehat{\chi})\right) \\
& =C(0,0)-C(\widehat{\chi} \otimes \widehat{\chi}),
\end{aligned}
$$

which shows that the above limit is finite. This concludes the proof of Lemma 1.

Recall that a function or distribution $u$ is said to be conditionally definite positive [13] on some space of functions $\mathcal{L}$ if $u(f * \tilde{f}) \geq 0$ whenever $f \in \mathcal{L}$. We are now able to state the following result.

\section{Theorem 3.}

An m.s continuous process $X$ on $\mathbb{R}$ has stationary nth increments if and only if its covariance has the form

$$
C(s, t)=r(s-t)+\sum_{j=0}^{n-1} s^{j} r_{j}(t)+t^{j} \overline{r_{j}}(s)
$$

where

i) $r$ is a symmetric continuous function, conditionally positive definite on $D^{n} \mathcal{S}(\mathbb{R}):=\left\{D^{n} \phi \mid\right.$ $\phi \in \mathcal{S}(\mathbb{R})\}$ and of growth $|r(x)| \leq O\left(1+|x|^{2 n}\right)$

ii) the $r_{j}$ are continuous polynomially bounded functions such that $\Re\left(\sum_{j=0}^{n-1} t^{j} r_{j}(t)\right) \geq 0$.

Proof. Suppose $C(s, t)$ has the form (3.5) and let the wavelet $g$ be in $\mathcal{S}_{\geq n}(\mathbb{R})$. Then the wavelet covariance

$$
C_{a}(s, t)=\widetilde{G}_{a} * C(s, t)=\int \frac{1}{a} \bar{g}\left(\frac{s^{\prime}-s}{a}\right) \frac{1}{a} g\left(\frac{t^{\prime}-t}{a}\right) r\left(s^{\prime}-t^{\prime}\right) d s^{\prime} d t^{\prime}
$$

is stationary since the contribution of the functions $r_{j}$ is cancelled by the $n$ vanishing moments of $g$. It follows from Theorem 2 that $X$ has stationary $n$th increments. Conversely, suppose $X$ has stationary $n$th increments. We generalize here a method that was first used in [35] to obtain a characterization of fractional Brownian motion by means of its wavelet transform. By Lemma 1 we have

$$
(-1)^{n} \xi^{n} \eta^{n} \widehat{C}(\xi, \eta)=d \mu(\xi) \delta(\xi+\eta)
$$

for some positive tempered measure $\mu$ with $\int\left(1+\xi^{2 n}\right)^{-1} d \mu(\xi)<\infty$. Under inverse Fourier transformation we obtain

$$
D_{s}^{n} D_{t}^{n} C(s, t)=F(s, t)
$$

for some $F \in \mathcal{S}^{\prime}\left(\mathbb{R}^{2}\right)$. Now let $\gamma \in \mathcal{S}^{\prime}(\mathbb{R})$ be the Fourier transform of the positive tempered measure $\mu$. By the Bochner-Schwartz theorem [37], $\gamma$ is a positive definite distribution [i.e., $\gamma(\psi * \tilde{\psi}) \geq 0$ for all $\psi \in \mathcal{S}(\mathbb{R})$ ] so it can be written as $\gamma=\left(1-\frac{D^{2}}{4 \pi}\right)^{k} u$ for some integer $k$ and some continuous positive definite function $u$. Now take $U$ a primitive of order $2 n$ of $u$ that we can choose to be symmetric [it suffices to set $u_{j+1}(x)=2^{-1}\left(\int_{0}^{x} u_{j}(t) d t+\int_{-x}^{0} \bar{u}_{j}(t) d t\right)$ for $j=0, .2 n-1$ with $u_{0}=u$ and $\left.U=u_{2 n}\right]$ and define $r=(-1)^{n}\left(1-\frac{D^{2}}{4 \pi}\right)^{k} U$. Then $r$ is a primitive of order $2 n$ of $(-1)^{n} \gamma$ and is symmetric [that is, $r(\tilde{\phi})=\overline{r(\phi)}$ for all $\phi \in \mathcal{S}(\mathbb{R})$ ], because the derivation of even order preserves the parity. Moreover, $r$ is by construction conditionally positive definite on $D^{n} \mathcal{S}(\mathbb{R})$ since $r\left(D^{n} \phi * \widetilde{D^{n} \phi}\right)=(-1)^{n} D^{2 n} r(\phi * \tilde{\phi})=\gamma(\phi * \tilde{\phi}) \geq 0$. Let us derive some 
regularity properties of $r$. Let $\chi \in C_{0}^{\infty}(\mathbb{R})$ be a positive cut-off function such that $\chi \geq 0$ and $\chi \equiv 1$ on $[-1,+1]$ and denote $\mu_{-}=\chi \mu$ and $\mu_{+}=(1-\chi) \mu$. Then both $\mu_{-}$and $\xi^{-2 n} \mu_{+}$are finite measures. Now let the distributions $\gamma_{-}$and $\gamma_{+}$be the Fourier transforms of $\mu_{-}$and $\mu_{+}$, respectively, and $r_{-}$and $r_{+}$primitives of order $2 n$ of $(-1)^{n} \gamma_{-}$and $(-1)^{n} \gamma_{+}$, respectively, that is $D^{2 n} r_{ \pm}=(-1)^{n} \gamma_{ \pm}$. On one hand $\gamma_{-}$, being the Fourier transform of a finite positive measure, is continuous and bounded and thus $r_{-}$is a continuous function of growth $\left|r_{-}(x)\right| \leq O\left(\left|x^{2 n}\right|\right)$. On the other hand, $\widehat{r_{+}}(\xi)=(-1)^{n} \xi^{-2 n} \mu_{+}(\xi)$ and thus $r_{+}$is the Fourier transform of a finite measure, and hence continuous and bounded. Since $D^{2 n} r=(-1)^{n} \gamma=(-1)^{n}\left(\gamma_{-}+\gamma_{+}\right)$, we have $r=r_{-}+r_{+}$ modulo a polynomial of degree $2 n$, which implies that $r$ is continuous with $|r(x)| \leq O\left(1+\left|x^{2 n}\right|\right)$. Now define $R(s, t)=r(s-t)$. Then for all $\phi, \psi \in \mathcal{S}(\mathbb{R})$ we have

$$
\begin{aligned}
D_{s}^{n} D_{t}^{n} R(\phi \otimes \psi) & =R\left(D^{n} \phi \otimes D^{n} \psi\right)=(-1)^{n} r\left(D^{2 n}(\phi * \widetilde{\psi})\right) \\
& =\gamma(\phi * \tilde{\psi})=\int d \mu(\xi) \widehat{\phi}(\xi) \widehat{\psi}(-\xi) \\
& =F(\phi \otimes \psi),
\end{aligned}
$$

and thus, by density of $\mathcal{S}(\mathbb{R}) \otimes \mathcal{S}(\mathbb{R})$ in $\mathcal{S}\left(\mathbb{R}^{2}\right), D_{s}^{n} D_{t}^{n} R=F$. To sum up, we have proven that

$$
D_{s}^{n} D_{t}^{n} C(s, t)=D_{s}^{n} D_{t}^{n} R(s, t),
$$

in $\mathcal{S}^{\prime}\left(\mathbb{R}^{2}\right)$ for a distribution $R$ with symmetric continuous kernel $r(s-t)$. Integrating once with respect each variable yields to

$$
D_{s}^{n-1} D_{t}^{n-1} C(s, t)-D_{s}^{n-1} D_{t}^{n-1} R(s, t)=\rho(t)+\sigma(s),
$$

for some distributions $\rho$ and $\sigma$ in $\mathcal{S}^{\prime}(\mathbb{R})$. Since the left-hand side is a Hermitian distribution [i.e., $\Sigma(t, s)=\overline{\Sigma(s, t)}]$ we must have $\sigma=\bar{\rho}$. Iterating this procedure, we obtain

$$
C(s, t)=r(s-t)+\sum_{j=0}^{n-1} s^{j} r_{j}(t)+t^{j} \overline{r_{j}}(s),
$$

for some tempered distributions $r_{j}$. The continuity of the functions $r_{j}$ follows from Lemma 6 in Appendix A. The condition $\mathfrak{A}\left(\sum_{j=0}^{n-1} t^{j} r_{j}(t)\right) \geq 0$ is forced by $C(t, t) \geq 0$. This concludes the proof of Theorem 3 .

Example 1. A well-known non-trivial example of process with stationary increments is fractional Brownian motion [23], $\mathrm{fBm}$ for short. For a given $H \in(0,1)$ (Hurst parameter), this is the unique zero-mean Gaussian process with covariance:

$$
C(s, t)=\frac{\sigma^{2}}{2}\left(|s|^{2 H}+|t|^{2 H}-|s-t|^{2 H}\right) .
$$

In this example clearly $r(x)=-r_{0}(x)=-\frac{\sigma^{2}}{2}|x|^{2 H}$. Note that $r$ is, as expected, conditionally positive definite on $D S(\mathbb{R})$ since for all $\varphi \in \mathcal{S}(\mathbb{R})$ :

$$
r\left(\varphi * \tilde{\varphi^{\prime}}\right)=\int \widehat{r}(\xi) \xi^{2}|\widehat{\varphi}(\xi)|^{2} d \xi=-C_{2 H} \int|\xi|^{1-2 H}|\widehat{\varphi}(\xi)|^{2} d \xi
$$

with $C_{2 H} \leq 0$ (see Appendix B). 


\section{Processes with Stationary Fractional Increments}

Wavelet Characterization. The results of Section 3 can be transferred to the fractional increments, provided the wavelet is taken in the spaces of the corresponding fractional moments. Any wavelet $h$ in the space $\mathcal{F}_{\geq \alpha}(\mathbb{R})$ is a $C^{\infty}$ function with the growth restriction:

$$
|h(t)| \leq O\left(\frac{1}{1+|t|^{\alpha+1-\epsilon}}\right),
$$

for $\epsilon>0$ arbitrarily small (this will be proven in Theorem 4). It follows that we will be able to define the wavelet transform of a process $X(t)$ with respect to a wavelet in $\mathcal{F}_{\geq \alpha}(\mathbb{R})$, provided the second moment satisfies

$$
\mathbf{H}_{\beta}: \mathbb{E} X(s) \overline{X(t)} \leq O\left(\left(1+|s|^{\beta}\right)\left(1+|t|^{\beta}\right)\right)
$$

for some $\beta<\alpha$. But first, we have to determine under which assumption the fractionally differentiated process is well defined.

Proposition 1.

Let $X(t)$ be a process on $\mathbb{R}$ satisfying $\mathbf{H}_{\beta}$. Then for all $\alpha>\beta$ and all $t \in \mathbb{R}$, the series

$$
\Delta_{\tau}^{\alpha} X(t):=\sum_{j=0}^{\infty} \pi_{j}^{\alpha} X(t-j \tau)
$$

is mean square convergent and the process $\Delta_{\tau}^{\alpha} X(t)$ satisfies again $\mathbf{H}_{\beta}$.

Proof. Let us check the Cauchy criterium for quadratic convergence. By hypothesis we have

$$
E|X(t-j \tau)|^{2} \leq O\left(1+|t-j \tau|^{2 \beta}\right) \leq O\left(\left(1+|t|^{2 \beta}\right)\left(1+|j|^{2 \beta}\right)\right) .
$$

Hence, for all integers $p \leq q$,

$$
\begin{aligned}
\mathbb{E}\left|\sum_{j=p}^{q} \pi_{j}^{\alpha} X(t-j \tau)\right|^{2} & =\sum_{j, k=p}^{q} \pi_{j}^{\alpha} \pi_{k}^{\alpha} \mathbb{E}(X(t-j \tau) \overline{X(t-k \tau)}) \\
& \leq \sum_{j, k=p}^{q}\left|\pi_{j}^{\alpha} \pi_{k}^{\alpha}\right|\left(\mathbb{E}|X(t-j \tau)|^{2} \mathbb{E}|X(t-k \tau)|^{2}\right)^{1 / 2} \\
& =\left(\sum_{j=p}^{q}\left|\pi_{j}^{\alpha}\right|\left(\mathbb{E}|X(t-j \tau)|^{2}\right)^{1 / 2}\right)^{2} \\
& \leq O\left(1+|t|^{2 \beta}\right)\left(\sum_{j=p}^{q}\left|\pi_{j}^{\alpha}\right|(1+|j|)^{\beta}\right)^{2} .
\end{aligned}
$$

Now Stirling's formula $\Gamma(x) \sim \sqrt{2 \pi} e^{-x+1}(x-1)^{x-1 / 2}$ as $x \rightarrow+\infty$, shows that $\left|\pi_{j}^{\alpha}\right| \sim j^{-\alpha-1}$ as $j \rightarrow+\infty$ and thus $\sum\left|\pi_{j}^{\alpha}\right|(1+|j|)^{\beta}<\infty$. It follows that the above partial sum vanishes as $p \rightarrow \infty$. This proves the mean square convergence of the series (4.2). Similarly, we obtain

$$
\mathbb{E}\left(\Delta_{\tau}^{\alpha} X(s) \overline{\Delta_{\tau}^{\alpha} X(t)}\right) \leq O\left(\left(1+|s|^{\beta}\right)\left(1+|t|^{\beta}\right)\right)
$$

and thus $\Delta_{\tau}^{\alpha} X$ satisfies again $\mathbf{H}_{\beta}$.

Next, we want to estimate the wavelet transform of $\Delta_{\tau}^{\alpha} X$.

\section{Lemma 2.}

Let $X$ be a process satisfying $\mathbf{H}_{\beta}$. Then for $\alpha>\beta$ and all $g \in \mathcal{S}(\mathbb{R})$ we have

$$
\left(\mathcal{W}_{g} \Delta_{\tau}^{\alpha} X\right)(b, a)=(\tau / a)^{\alpha}\left(\mathcal{W}_{\left.h_{\{\tau} / a\right]} X\right)(b, a) a . s,
$$


where the wavelet $h_{[\tau]}$, defined by

$$
h_{[\tau]}(t)=\tau^{-\alpha} \sum_{j=0}^{\infty} \pi_{j}^{\alpha} g(t-\tau j)
$$

satisfies

$$
\sup _{\tau>0}\left|h_{[\tau]}(t)\right| \leq O\left(\frac{1}{1+|t|^{\alpha+1-\epsilon}}\right)
$$

for all $\epsilon>0$, and

$$
\widehat{h_{[\tau]}}(\xi) \sim|\xi|^{\alpha} e^{i \frac{\alpha \pi}{2} \operatorname{sign}(\xi)} \widehat{g}(\xi), \xi \rightarrow 0
$$

Proof. First we state

$$
\int g_{b a}(t) \Delta_{\tau}^{\alpha} X(t) d t=\sum_{j=0}^{\infty} \pi_{j}^{\alpha} \int g_{b a}(t) B_{\tau}^{j} X(t) d t \text { a.s } .
$$

Indeed, by Fatou's lemma and the majorizations of the previous lemma,

$$
\begin{aligned}
& \mathbb{E}\left|\int g_{b a}(t) \Delta_{\tau}^{\alpha} X(t) d t-\sum_{j=0}^{\infty} \pi_{j}^{\alpha} \int g_{b a}(t) B_{\tau}^{j} X(t) d t\right| \\
= & \mathbb{E} \liminf _{p \rightarrow \infty}\left|\int g_{b a}(t) \Delta_{\tau}^{\alpha} X(t) d t-\sum_{j=0}^{p} \pi_{j}^{\alpha} \int g_{b a}(t) B_{\tau}^{j} X(t) d t\right| \\
= & \mathbb{E} \liminf _{p \rightarrow \infty}\left|\int g_{b a}(t)\left[\Delta_{\tau}^{\alpha} X(t)-\sum_{j=0}^{p} \pi_{j}^{\alpha} B_{\tau}^{j} X(t)\right] d t\right| \\
\leq & \liminf _{p \rightarrow \infty} \mathbb{E}\left|\int g_{b a}(t)\left[\Delta_{\tau}^{\alpha} X(t)-\sum_{j=0}^{p} \pi_{j}^{\alpha} B_{\tau}^{j} X(t)\right] d t\right| \\
\leq & \liminf _{p \rightarrow \infty} \int\left|g_{b a}(t)\right| \mathbb{E}\left|\Delta_{\tau}^{\alpha} X(t)-\sum_{j=0}^{p} \pi_{j}^{\alpha} B_{\tau}^{j} X(t)\right| d t \\
\leq & \liminf _{p \rightarrow \infty} \int\left|g_{b a}(t)\right|\left(1+|t|^{\beta}\right) d t \sum_{j=p+1}^{\infty} \pi_{j}^{\alpha}(1+|j|)^{\beta}=0 .
\end{aligned}
$$

Since by a simple change of variables we have

$$
\left(\mathcal{W}_{g} B_{\tau}^{j} X\right)(b, a)=\left(\mathcal{W}_{g-j \tau / a, 1} X\right)(b, a)
$$

where $g-j \tau / a, 1(t)=g(t+j \tau / a)$, it follows that

$$
\left(\mathcal{W}_{g} \Delta_{\tau}^{\alpha} X\right)(b, a)=\sum_{j=0}^{\infty} \pi_{j}^{\alpha}\left(\mathcal{W}_{g_{-j \tau / a, 1}} X\right)(b, a) \text { a.s }
$$

The form of the right-hand side suggests the introduction of the function

$$
h_{[\tau]}=\tau^{-\alpha}\left(\sum_{j=0}^{\infty} \pi_{j}^{\alpha} \delta_{j \tau}\right) * g,
$$

$\delta_{\lambda}$ being the delta function centered at $\lambda$. Its Fourier transform is given by

$$
\widehat{h}_{[\tau]}(\xi)=\tau^{-\alpha}\left(\sum_{j=0}^{\infty} \pi_{j}^{\alpha} e^{-i j \tau \xi}\right) \widehat{g}(\xi)
$$


Now consider the function of the complex variable $z$

$$
(1-z)^{\alpha}:=\left\{\begin{array}{l}
\exp (\alpha \log (1-z)) \text { if }|z| \leq 1, z \neq 1, \\
0 \text { if } z=1
\end{array}\right.
$$

where $\log z$ is the principal branch of the complex $\operatorname{logarithm}$, i.e., $\log \left(\rho e^{i \theta}\right)=\log \rho+i \theta,-\pi<$ $\theta<\pi, \rho \neq 0$. This function is analytic in the disk $|z|<1$ and for $|z| \leq 1$ one can show that

$$
(1-z)^{\alpha}=\sum_{j=0}^{\infty} \pi_{j}^{\alpha} z^{j}
$$

It follows that

$$
\widehat{h}_{[\tau]}(\xi)=\tau^{-\alpha}\left(1-e^{-i \tau \xi}\right)^{\alpha} \widehat{g}(\xi)
$$

The factor $\left(1-e^{-i \tau \xi}\right)^{\alpha}$ is a continuous function (in fact $C^{\infty}$ except at the points $2 \pi k / \tau, k \in \mathbb{N}$, where it is only Lipschitz of order $\alpha$ ), with the following behavior at the origin:

$$
\tau^{-\alpha}\left(1-e^{-i \tau \xi}\right)^{\alpha} \sim|\xi|^{\alpha} e^{i \frac{\alpha \pi}{2} \operatorname{sign}(\xi)}, \xi \rightarrow 0
$$

Moreover,

$$
\sup _{\tau>0}\left|h_{[\tau]}(t)\right| \leq O\left(\frac{1}{1+|t|^{\alpha+1-\epsilon}}\right)
$$

for $\epsilon$ arbitrarily small. This last estimation is technical and has been relegated to Appendix A (Lemma 8). Note that, in spite of its asymptotics, $h_{[\tau]}$ is not in $\mathcal{F}_{\geq \alpha}(\mathbb{R})$ because of the singularities of the function $\left(1-e^{-i \tau \xi}\right)^{\alpha}$. To conclude the proof, we only need to show that

$$
\sum_{j=0}^{\infty} \pi_{j}^{\alpha}\left(\mathcal{W}_{g_{-j \tau / a, 1}} X\right)(b, a)=(\tau / a)^{\alpha} \mathcal{W}_{h_{[\tau / a]}} X(b, a) \text { a.s }
$$

The problem is to permute summation and wavelet transform. For this, let

$$
h_{[\tau]}^{p}(t)=\tau^{-\alpha} \sum_{j=p+1}^{\infty} \pi_{j}^{\alpha} g(t-j \tau)
$$

for which

$$
\sup _{p \geq 1}\left|h_{[\tau]}^{p}(t)\right| \leq \tau^{-\alpha} \sum_{j=1}^{\infty} \pi_{j}^{\alpha}|g(t-j \tau)| \leq C_{\tau, \epsilon} \frac{1}{1+|t|^{\alpha+1-\epsilon}}
$$

with $\epsilon$ arbitrarily small (see Lemma 8 ). Then by Fatou's lemma we again have

$$
\begin{aligned}
& \mathbb{E}\left|\sum_{j=0}^{\infty} \pi_{j}^{\alpha}\left(\mathcal{W}_{g_{-j \tau / a, 1}} X\right)(b, a)-(\tau / a)^{\alpha} \mathcal{W}_{h_{[\tau / a]}} X(b, a)\right| \\
= & \mathbb{E} \liminf _{p \rightarrow \infty}\left|\sum_{j=0}^{p} \pi_{j}^{\alpha}\left(\mathcal{W}_{g-j \tau / a, 1} X\right)(b, a)-(\tau / a)^{\alpha} \mathcal{W}_{h_{[\tau / a]}} X(b, a)\right| \\
= & (\tau / a)^{\alpha} \mathbb{E} \liminf _{p \rightarrow \infty}\left|\left(\mathcal{W}_{h_{[\tau / a]}^{p}} X\right)(b, a)\right| \\
\leq & (\tau / a)^{\alpha} \liminf _{p \rightarrow \infty} \mathbb{E} \int \frac{1}{a}\left|h_{[\tau / a]}^{p}\left(\frac{t-b}{a}\right)\right| \mathbb{E}|X(t)| d t .
\end{aligned}
$$


Now for fixed $t, h_{[\tau / a]}^{p}(t) \rightarrow 0$ as $p \rightarrow \infty$. On the other hand, the integrand is uniformly bounded by an integrable function in view of (4.11) and $\mathbf{H}_{\beta}$. Hence, the Lebesgue dominated convergence theorem applies and the above limit is zero. Equation (4.3) follows by combining (4.7) and (4.10).

Now we can state the following result.

\section{Theorem 4.}

Let $X$ be an m.s continuous process on $\mathbb{R}$ satisfying $\mathbf{H}_{\beta}$ and let $\alpha>\beta$. Then the wavelet transform $\mathcal{W}_{h} X(\cdot, a)$ is well defined for all wavelet $h$ in $\mathcal{F}_{\geq \alpha}(\mathbb{R})$ and we have the following properties:

i) If $X$ has stationary $\alpha$ th increments, then the wavelet process $\mathcal{W}_{h} X(\cdot, a)$ is stationary for all wavelet $h$ in $\mathcal{F}_{\geq \alpha}(\mathbb{R})$ and for all $a>0$.

ii) Conversely, if for some wavelet $h$ in $\mathcal{F}_{=\alpha}(\mathbb{R}), \mathcal{W}_{h} X(\cdot, a)$ is stationary for all $a>0$, then $X$ has stationary $\alpha$ th increments.

iii) If furthermore $\widehat{h}(\xi) \neq 0$ for $\xi \neq 0$, then it is enough that $\mathcal{W}_{h} X(\cdot, a)$ be stationary for some $a>0$, for $X$ to have stationary $\alpha$ th increments.

Proof. i) Let $h \in \mathcal{F}_{\geq \alpha}(\mathbb{R})$ be given by

$$
\widehat{h}(\xi)=e^{i \frac{\alpha \pi}{2} \operatorname{sign}(\xi)}|\xi|^{\alpha} \widehat{g}(\xi),
$$

for some $g \in \mathcal{S}(\mathbb{R})$ and $h_{[\tau]}$ defined by (4.8). Then it is easy to verify that $\left\|\widehat{h}_{[\tau]}-\widehat{h}\right\|_{1} \rightarrow 0$ as $\tau \rightarrow 0$, and hence $\left\|h_{[\tau]}-h\right\|_{\infty} \rightarrow 0$ as $\tau \rightarrow 0$. It follows from (4.9) that

$$
|h(t)| \leq O\left(\frac{1}{1+|t|^{\alpha+1-\epsilon}}\right),
$$

for $\epsilon$ arbitrary small, and thus under condition $\mathbf{H}_{\beta}$ the wavelet transform is well defined. Now let

$$
\begin{aligned}
& C_{a}(s, t)=\mathbb{E} \mathcal{W}_{h} X(s, a) \overline{\mathcal{W}_{h} X(t, a)}-\mathbb{E} \mathcal{W}_{h} X(s, a) \mathbb{E} \overline{\mathcal{W}_{h} X(t, a)}, \\
& C_{a}^{\tau}(s, t)=\mathbb{E} \mathcal{W}_{h_{[\tau]}} X(s, a) \overline{\mathcal{W}_{h_{[\tau]}} X(t, a)}-\mathbb{E} \mathcal{W}_{h_{[\tau]}} X(s, a) \mathbb{E} \overline{\mathcal{W}_{h_{[\tau]}} X(t, a)},
\end{aligned}
$$

be the wavelet covariances computed w.r.t $h$ and $h_{[\tau]}$, respectively. Since $\sup _{\tau>0}\left|h_{[\tau]}(t)\right| \leq$ $O\left(1+|t|^{\alpha+1-\epsilon}\right)^{-1}$, we may apply Lebesgue dominated convergence theorem to prove that, for all fixed $s$ and $t$,

$$
\begin{aligned}
\lim _{\tau \rightarrow 0} C_{a}^{\tau}(s, t) & =\lim _{\tau \rightarrow 0} \int \frac{1}{a} \overline{h_{[\tau]}}\left(\frac{s^{\prime}-s}{a}\right) \frac{1}{a} h_{[\tau]}\left(\frac{t^{\prime}-t}{a}\right) C\left(s^{\prime}, t^{\prime}\right) d s^{\prime} d t^{\prime} \\
& =\int \frac{1}{a} \bar{h}\left(\frac{s^{\prime}-s}{a}\right) \frac{1}{a} h\left(\frac{t^{\prime}-t}{a}\right) C\left(s^{\prime}, t^{\prime}\right) d s^{\prime} d t^{\prime} \\
& =C_{a}(s, t) .
\end{aligned}
$$

Now by Lemma $2, \mathcal{W}_{h_{[\tau]}} X(b, a)=\tau^{-\alpha}\left(\mathcal{W}_{g} \Delta_{a \tau}^{\alpha} X\right)(b, a)$, which is stationary by Theorem 1 . Therefore, all the covariances $C_{a}^{\tau}(s, t)$ are stationary. But then the limit $C_{a}(s, t)$ must also be stationary. The same reasoning applies to the mean, proving the first statement.

ii)+iii) Now suppose the function $g$ in (4.12) is in $\mathcal{S}_{0}(\mathbb{R})$ (which is equivalent to $h$ being in $\mathcal{F}_{=\alpha}(\mathbb{R})$ ). For the proof, we will need some auxiliary functions. Let $\chi$ be a function in $\mathcal{S}_{0}(\mathbb{R})$ such that $\widehat{\chi}(\xi) \neq 0$ for all $\xi, 0 \leq \varphi \leq 1$ another function in $C_{0}^{\infty}(\mathbb{R})$ such that $\varphi(\xi)=1$ for $|\xi| \leq 1 / 2$ and $\varphi(\xi)=0$ for $|\xi| \geq 1$, and $\varphi^{\lambda}=\lambda(1-\varphi)+\varphi$. Finally, for all $0<\lambda<1$, and for some fixed $\tau>0$ define

$$
\widehat{s_{[\lambda, \tau]}}(\xi)=\tau^{-\alpha}\left[1-\varphi^{\lambda}(\tau \xi) e^{-i \tau \xi}\right]^{\alpha} \widehat{g}(\xi)
$$


Note that the term [ $]^{\alpha}$ is well defined since $\left|\varphi^{\lambda}\right| \leq 1$. Now let us rewrite

$$
\widehat{\chi}(\tau \xi) \widehat{h}(\xi)=\widehat{s[\lambda, \tau]}(\xi)|\tau \xi|^{\alpha} e^{i \frac{\alpha \pi}{2} \operatorname{sign}(\tau \xi)}\left[1-\varphi^{\lambda}(\tau \xi) e^{-i \tau \xi}\right]^{-\alpha} \widehat{\chi}(\tau \xi)=: \widehat{s_{[\lambda, \tau]}}(\xi) \widehat{\phi}(\tau \xi),
$$

with

$$
\widehat{\phi}(\xi)=|\xi|^{\alpha} e^{i \frac{\alpha \pi}{2} \operatorname{sign}(\xi)}\left[1-\varphi^{\lambda}(\xi) e^{-i \xi}\right]^{-\alpha} \widehat{\chi}(\xi)=\left\{\begin{array}{l}
|\xi|^{\alpha} e^{i \frac{\alpha \pi}{2} \operatorname{sign}(\xi)}\left[1-e^{-i \xi}\right]^{-\alpha} \widehat{\chi}(\xi),|\xi| \leq 1 / 2, \\
|\xi|^{\alpha} e^{i \frac{\alpha \pi}{2} \operatorname{sign}(\xi)}\left[1-\lambda e^{-i \xi}\right]^{-\alpha} \widehat{\chi}(\xi),|\xi| \geq 1
\end{array}\right.
$$

For $|\xi|>1$, the function $\widehat{\phi}$ is clearly $C^{\infty}$, rapidly decreasing and non-vanishing. For $|\xi| \leq 1$, the only possible singularity might be at zero. Now using $(i \xi)^{\alpha}=|\xi|^{\alpha} e^{i \frac{\alpha \pi}{2} \operatorname{sign}(\xi)}$ we observe that

$$
\left(1-e^{-i \xi}\right)^{\alpha}|\xi|^{-\alpha} e^{-i \frac{\alpha \pi}{2} \operatorname{sign}(\xi)}=\left(1+\sum_{j=2}^{\infty} \frac{(-i \xi)^{j-1}}{j !}\right)^{\alpha}
$$

which in the neighborhood of the origin is a smooth function bounded away from zero [since $\left|\sum_{j=2}^{\infty} \frac{(-i \xi)^{j-1}}{j !}\right| \leq|\exp | \xi|-1|<1$ whenever $\left.|\xi| \leq 1 / 2\right]$. Altogether this shows that $\phi$ is a welldefined function in $\mathcal{S}(\mathbb{R})$ such that $\widehat{\phi}$ never vanishes. Now by Theorem 1 i), $\mathcal{W}_{h} X(\cdot, a)=\tilde{h}_{a} * X$ stationary for all (some) $a>0$ implies $\mathcal{W}_{\chi_{\tau} * h} X(\cdot, a)=\left(\tilde{\chi}_{\tau}\right)_{a} *\left(\tilde{h}_{a} * X\right)$ stationary for all (some) $a>0$, that is $\mathcal{W}_{\phi_{\tau} * s[\lambda, \tau]} X(\cdot, a)$ is stationary for all (some) $a>0$. Since $\widehat{\phi_{\tau}}$ never vanishes, this entails (Theorem 1 iii) that $\mathcal{W}_{s_{[\lambda, \tau]}} X(\cdot, a)$ is stationary for all (some) $a>0$. Now we have the pointwise convergence $s_{[\lambda, \tau]}(\xi) \rightarrow h_{[\tau]}(\xi), \lambda \rightarrow 1$ [because $\left\|s_{[\lambda, \tau]}-h_{[\tau]}\right\|_{\infty} \leq\left\|\widehat{s_{[\lambda, \tau]}}-\widehat{h_{[\tau]}}\right\|_{1} \rightarrow 0$ ] and the $s_{[\lambda, \tau]}$ are uniformly bounded (w.r.t $\lambda$ ) by $(2 / \tau)^{\alpha}\|g\|_{1}$. Hence, we may apply the Lebesgue dominated convergence theorem to conclude, as before, that the wavelet covariance computed w.r.t the wavelet $s_{[\lambda, \tau]}$ converge to the one computed w.r.t the wavelet $h_{[\tau]}$ as $\lambda \rightarrow 1$. The same holds for the mean. Therefore, we have shown that $\mathcal{W}_{h[\tau]} X(\cdot, a)$ is stationary for all (some) $a>0$, whenever $\mathcal{W}_{h} X(\cdot, a)$ is stationary for all (some) $a>0$. Applying this to $\tau / a$ rather than $\tau$, we obtain (Lemma 2) that $\mathcal{W} g \Delta_{\tau}^{\alpha} X$ is stationary for all (some) $a>0$, which by Theorem 1 ii (iii if $\widehat{g} \neq 0$ ) implies that $\Delta_{\tau}^{\alpha} X$ is stationary. This concludes the proof.

Remark. A result analogous to the statement $\mathrm{i}$ ) of Theorem 4 has already been given for the discrete wavelet coefficients of a multiresolution analysis [20] and wavelet packets decomposition [30].

Application to Fractional Brownian Motion. The previous theorem leads to a direct proof of the stationarity of the fractional increments of $\mathrm{fBm}$ (a result that was first proved in [29], [31]).

\section{Theorem 5.}

Fractional Brownian motion with Hurst exponent $H$ has $\alpha$-stationary increments for any $\alpha>H$.

Proof. Recall that the covariance of $\mathrm{fBm}$ is given by

$$
C(s, t)=\frac{\sigma^{2}}{2}\left(|s|^{2 H}+|t|^{2 H}-|s-t|^{2 H}\right)
$$

The bi-dimensional Fourier transform of this equation is

$$
\widehat{C}(\xi, \eta)=-\frac{\sigma^{2}}{2} C_{2 H}\left(-|\xi|^{-1-2 H} \delta(\eta)-|\eta|^{-1-2 H} \delta(\xi)+|\xi|^{-1-2 H} \delta(\xi+\eta)\right),
$$


where the definition of the distribution $|\xi|^{-1-2 H}$ is recalled in Appendix $\mathrm{B}$, and $C_{2 H}$ is a negative constant. Now let $g$ be a wavelet in $\mathcal{F}_{=\alpha}(\mathbb{R})$ for some $\alpha>H$. Then

$$
\begin{aligned}
\widehat{C_{a}}(\xi, \eta) & =\widehat{\widehat{g}(a \xi)} \widehat{g}(-a \eta) \widehat{C}(\xi, \eta) \\
& =-\frac{\sigma^{2}}{2} C_{2 H} \overline{\widehat{g}(a \xi)} \widehat{g}(-a \eta)\left(-|\xi|^{-1-2 H} \delta(\eta)-|\eta|^{-1-2 H} \delta(\xi)+|\xi|^{-1-2 H} \delta(\xi+\eta)\right) .
\end{aligned}
$$

Since $|\widehat{g}(\xi)| \sim|\xi|^{\alpha}, \xi \rightarrow 0$, the first two terms on the right-hand side vanish and it remains

$$
\left.\widehat{C_{a}}(\xi, \eta)=-\frac{\sigma^{2}}{2} C_{2 H}|\widehat{g}(a \xi)|^{2}|\xi|^{-1-2 H} \delta(\xi+\eta)\right)=: d \mu_{a}(\xi) \delta(\xi+\eta) .
$$

Now $|\widehat{g}(a \xi)|^{2}|\xi|^{-1-2 H}=\phi(\xi)|\xi|^{2 \alpha-1-2 H}$ with $\phi(\xi)=|\widehat{g}(a \xi)|^{2}|\xi|^{-2 \alpha} \in \mathcal{S}(\mathbb{R})$ and $2 \alpha-1-$ $2 H>-1$. Thus, the measure $\mu_{a}$ is positive and finite. It follows from Lemma 4 that $C_{a}$ is a stationary covariance. But in view of Theorem 4 this in turn implies that the process has stationary $\alpha$-increments.

\section{Locally Stationary Processes}

In this section, we will abandon hypothesis $\mathbf{H}$ and instead require that the process $X(t)$ be harmonizable. Recall that a process is strongly harmonizable or harmonizable in the sense of Loève [21] if there is a random process $Y(\xi)$ with a covariance of bounded variation such that $X(t)=\int e^{i t \xi} d Y(\xi)$ a.s., which is equivalent to the existence of a complex Borel measure $M$ on $\mathbb{R}^{2}$ such that

$$
C(s, t)=\int e^{-i(s \xi-t \eta)} d M(\xi, \eta) .
$$

Such covariances are also said to be (strongly) harmonizable. To put it differently, a process is strongly harmonizable if and only if its covariance is the Fourier transform of a measure of bounded variations on $\mathbb{R}^{2}$. This implies in particular that the covariance is continuous and bounded. Hence, the strong harmonizability condition supplements and strengthens condition $\mathbf{H}$. Recall also that a process is weakly harmonizable or harmonizable in the sense of Rozanov [36] if (5.1) holds only for a complex bi-measure on $\mathbb{R}^{2}$ (i.e., separately $\sigma$-additive on the Cartesian product $\mathcal{B}(\mathbb{R}) \times \mathcal{B}(\mathbb{R})$ of the Borel algebras.) We refer to [18] for further details on the different notions of harmonizability and other spectral properties. The wavelet transform is known to preserve weak- and strongharmonizability [5]. However, we will restrict ourselves to the latter property and harmonizability will be understood throughout in the strong sense.

A covariance $C(s, t)$ is locally stationary if it can be written

$$
C(s, t)=m\left(\frac{s+t}{\sqrt{2}}\right) \gamma\left(\frac{s-t}{\sqrt{2}}\right)
$$

for some positive function $m$ and some covariance function $\gamma$. As was shown in [27], a locally stationary process is harmonizable if and only if $\gamma$ is the Fourier transform of a probability measure and $m$ is the Fourier transform of a finite complex measure. This can be seen as a generalization of Khintchine theorem. Simple examples of harmonizable locally stationary covariances are given by the following family of functions.

Example 2. Any function of the form $C(s, t)=\exp \left(-\alpha(s+t)^{2}\right) c(s-t) \exp \left(-\alpha(s-t)^{2}\right)$ where $\alpha>0$ and where $c$ is a stationary covariance is a harmonizable locally stationary covariance. 
Proof. The locally stationary structure is plain. Moreover, $C(s, t)$ is positive definite since for all functions $\varphi \in \mathcal{S}(\mathbb{R})$ we have

$$
\int C(s, t) \bar{\varphi}(s) \varphi(t) d s d t=\int c(s-t) \bar{\varphi}(s) \exp \left(-2 \alpha s^{2}\right) \varphi(t) \exp \left(-2 \alpha t^{2}\right) d s d t \geq 0 .
$$

Finally $C(s, t)$ is harmonizable since $m(t)=\exp \left(-\alpha t^{2}\right)$ is the Fourier transform of a Gaussian function (hence a finite measure) while $\gamma(t)=c(t) \exp \left(-\alpha t^{2}\right)$ is the Fourier transform of the positive finite measure $\mu * \chi$, where $\widehat{\mu}=c$ and $\chi$ is a Gaussian function.

The form of the covariance suggests the introduction of the following new notation. We define the "square product" $\otimes$ of two functions in $\mathcal{S}(\mathbb{R})$ by

$$
(\phi \otimes \psi)(s, t)=\phi\left(\frac{s+t}{\sqrt{2}}\right) \psi\left(\frac{s-t}{\sqrt{2}}\right) .
$$

The square product is a bilinear operation from $\mathcal{S}(\mathbb{R}) \times \mathcal{S}(\mathbb{R})$ to $\mathcal{S}\left(\mathbb{R}^{2}\right)$ possessing the following elementary properties with respect to the Fourier transform and the tensor product:

$$
\begin{aligned}
\widehat{\phi \otimes \psi} & =\widehat{\phi} \otimes \widehat{\psi}, \\
(\phi \otimes \psi)\left(\frac{s+t}{\sqrt{2}}, \frac{s-t}{\sqrt{2}}\right) & =(\phi \otimes \psi)(s, t) .
\end{aligned}
$$

We define the square product of two tempered measures $\mu$ and $\nu$ of $\mathcal{S}^{\prime}(\mathbb{R})$ by duality:

$$
(\mu \otimes \nu)(\Phi)=\int d \mu(\xi) d \nu(\eta) \Phi\left(\frac{\xi+\eta}{\sqrt{2}}, \frac{\xi-\eta}{\sqrt{2}}\right) .
$$

In general, the wavelet transform of locally stationary processes is no longer locally stationary. However, there exists a class of filters, namely the Gaussian filters, for which the local stationarity is conserved. Indeed, any function of the form $g(x)=\alpha e^{-\beta x^{2}}$ has the property that

$$
g(s) g(t)=g\left(\frac{s+t}{\sqrt{2}}\right) g\left(\frac{s-t}{\sqrt{2}}\right) .
$$

If $X$ is a process with covariance $C(s, t)=m(s+t) \gamma(s-t)$, then a straightforward calculation using (5.4) shows that

$$
C_{a}(s, t)=g_{a} * m\left(\frac{s+t}{\sqrt{2}}\right) g_{a} * \gamma\left(\frac{s-t}{\sqrt{2}}\right)
$$

and thus the wavelet process for the Gaussian wavelets is locally stationary as soon as the process itself is locally stationary.

The converse is also true. To show it, we need the following lemma.

\section{Lemma 3.}

i) If $C(s, t)$ is a locally stationary harmonizable covariance function, then its Fourier transform has the form $\widehat{C}=\mu \otimes v$ for some finite complex, resp. positive, measures $\mu$ and $v$.

ii) Conversely, if $C(s, t)$ is a polynomially bounded continuous covariance function with a Fourier transform of the above form, where $\mu$ and $\nu$ are a priori only tempered measures and $v$ positive, then $C(s, t)$ is locally stationary.

Proof. i) Suppose $C=m \otimes \gamma$ is a locally stationary harmonizable covariance and let $\mu$ and $\nu$ be the Fourier transforms of the functions $m$ and $\gamma$. By hypothesis, $\nu$ is a finite positive measure and $\mu$ is a finite complex measure. Now if $\Phi$ is a test function in $\mathcal{S}\left(\mathbb{R}^{2}\right)$, then

$$
\begin{aligned}
\widehat{C}(\widehat{\Phi}) & =\int m\left(\frac{s+t}{\sqrt{2}}\right) \gamma\left(\frac{s-t}{\sqrt{2}}\right) \Phi(-s,-t) d s d t \\
& =\int m(u) \gamma(v) \Psi(-u,-v) d u d v \\
& =(\mu \otimes \nu)(\widehat{\Psi}),
\end{aligned}
$$


where we have performed the bijective change of variables $u=(s+t) / \sqrt{2}, v=(s-t) / \sqrt{2}$, and set $\Psi(s, t)=\Phi\left(\frac{s+t}{\sqrt{2}}, \frac{s-t}{\sqrt{2}}\right)$. Now $\widehat{\Psi}(\xi, \eta)=\widehat{\Phi}\left(\frac{\xi+\eta}{\sqrt{2}}, \frac{\xi-\eta}{\sqrt{2}}\right)$ and thus $\widehat{C}(\widehat{\Phi})=(\mu \otimes \nu)(\widehat{\Phi})$, which proves i).

ii) Assume $\widehat{C}$ has the form $\widehat{C}=\mu \otimes v$ with $\mu$ and $v$ a priori only tempered measures and $v$ positive and let the distributions $m$ and $\gamma$ be their respective Fourier transforms. For any $\phi, \psi$ in $\mathcal{S}(\mathbb{R})$ we have

$$
\begin{aligned}
\widehat{C}(\widehat{\phi \otimes \psi}) & =\int d \mu(\xi) d \nu(\eta) \widehat{\phi \otimes \psi}\left(\frac{\xi+\eta}{\sqrt{2}}, \frac{\xi-\eta}{\sqrt{2}}\right) \\
& =\mu(\widehat{\phi}) \nu(\widehat{\psi})=m(\phi) \gamma(\psi) .
\end{aligned}
$$

Now take an approximate identity $\phi_{a}$ in $\mathcal{S}^{\prime}(\mathbb{R})$. Then

$$
\begin{aligned}
\lim _{a \rightarrow 0} \widehat{C}\left(\widehat{\phi_{b a} \otimes \psi}\right) & =\lim _{a \rightarrow 0} \int C(s, t)\left(\phi_{b a} \otimes \psi\right)(-s,-t) d s d t \\
& =\lim _{a \rightarrow 0} \int C\left(\frac{s+t}{\sqrt{2}}, \frac{s-t}{\sqrt{2}}\right) \phi_{b a}(-s) \psi(-t) d s d t \\
& =\int C\left(\frac{-b+t}{\sqrt{2}}, \frac{-b-t}{\sqrt{2}}\right) \psi(-t) d t=: C_{b}(\psi) .
\end{aligned}
$$

On the other hand, since $\widehat{C}\left(\widehat{\phi_{b a} \otimes} \psi\right)=m\left(\phi_{b a}\right) \gamma(\psi)$, this shows, first, that the limit $\lambda_{b}=\lim _{a \rightarrow 0}$ $m\left(\phi_{b a}\right)$ exists and, second, that $\gamma(\psi)=\lambda_{b}^{-1} C_{b}(\psi)$, which implies that $\gamma$ is a continuous function. We need only to verify that $\lambda_{b} \neq 0$ for at least one $b$. Now since $m\left(\phi_{b a}\right) \rightarrow m(b+\cdot)$ in $\mathcal{S}^{\prime}(\mathbb{R})$, $\lambda_{b}=0$ for all $b$ would imply $m=0$ and $C(s, t) \equiv 0$, in which case the lemma is trivially satisfied. Exchanging the functions $\phi_{b a}$ and $\psi$, we can show in the same way that $m$ is a continuous function. Now for any $b, b^{\prime}$ we have

$$
\begin{aligned}
C\left(\frac{b+b^{\prime}}{\sqrt{2}}, \frac{b-b^{\prime}}{\sqrt{2}}\right) & \left.=\lim _{a \rightarrow 0} \int C\left(\frac{s+t}{\sqrt{2}}, \frac{s-t}{\sqrt{2}}\right) \phi_{b a}(s) \phi_{b^{\prime} a}\right)(t) d s d t \\
& =\lim _{a \rightarrow 0} \int C(s, t)\left(\phi_{b a} \otimes \phi_{b^{\prime} a}\right)(s, t) d s d t \\
& =\lim _{a \rightarrow 0} m\left(\phi_{b a}\right) \gamma\left(\phi_{b^{\prime} a}\right) \\
& =m(b) \gamma\left(b^{\prime}\right),
\end{aligned}
$$

which shows that $C=m \otimes \gamma$. It remains to show that $\gamma$ is a stationary covariance. For this, it is enough to show that $\nu$ is a finite positive measure. Now take an approximate identity $\phi_{a}$ in $\mathcal{S}(\mathbb{R})$.

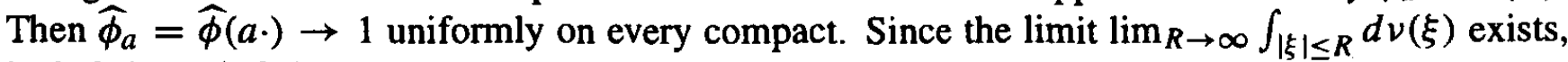
be it finite or infinite, we have

$$
\int d \nu(\xi)=\lim _{a \rightarrow 0} \nu\left(\widehat{\phi}_{a}\right)=\lim _{a \rightarrow 0} \gamma\left(\widehat{\hat{\phi}_{a}}\right)=\gamma(0),
$$

which shows that $v$ is finite. This concludes the proof of the lemma.

Now we can prove the following result.

\section{Theorem 6.}

Let $X$ be an m.s continuous process satisfying $\mathbf{H}$ and $g(x)=\alpha \exp \left(-\beta x^{2}\right)$ a Gaussian function.

i) If $X$ is locally stationary, then $\mathcal{W}_{g} X(\cdot, b)$ is locally stationary for all $a>0$.

ii) Conversely, if $\mathcal{W}_{g} X(\cdot, a)$ is locally stationary and harmonizable for some $a>0$, then $X$ is locally stationary.

Proof. Only ii) is to be proved. If the wavelet process is locally stationary and harmonizable, then

$$
\widehat{C_{a}}(\xi, \eta)=\widehat{g}(a \xi) \widehat{g}(-a \eta) \widehat{C}(\xi, \eta)=d \mu_{a}(\xi+\eta) d v_{a}(\xi-\eta),
$$

for some finite complex, resp. positive, measures $\mu_{a}$ and $v_{a}$. Thus

$$
\widehat{C}(\xi, \eta)=\frac{\alpha^{2}}{2 \beta} e^{\frac{a^{2}}{8 \beta}(\xi+\eta)^{2}} e^{\frac{a^{2}}{8 \beta}(\xi-\eta)^{2}} d \mu_{a}(\xi+\eta) d v_{a}(\xi-\eta)=: d \sigma_{a}(\xi+\eta) d \theta_{a}(\xi-\eta) .
$$


The above equality holds a priori in $\mathcal{D}^{\prime}\left(\mathbb{R}^{2}\right)$. However, the left-hand side is a tempered distribution and thus the right-hand side is a tempered measure on $\mathbb{R}^{2}$. By Lemma 5 ii), this implies that $\sigma_{a}$ and $\theta_{a}$ are themselves tempered measures on $\mathbb{R}$, with $\theta_{a}$ positive. The conclusion follows from Lemma 3 .

Remark: The family of Gaussian wavelets is in fact the only one which can characterize locally stationary processes since it was shown in [28] that the only smooth filters on the spectral measure preserving local stationarity are the Gaussian filters.

\section{Conclusion}

We have seen that the continuous wavelet transform is an appropriate tool to characterize a large class of non-stationary processes, provided the analyzing wavelet is suitably chosen. Two applications have been proposed: the derivation of the covariance structure of processes with stationary $n$th increments and the stationarity of the fractional increments of fractional Brownian motion. However, some questions remain open, such as how to choose $a$ priori the functions $r$ and $r_{j}$ in (3.5) to form a covariance function or if there is an analogous version of Theorem 3 for the stationary fractional increments.

\section{Acknowledgments}

I am very grateful to Holger Rootzén for his advice and encouragement. Many thanks also to Jöran Bergh, Matthias Holschneider, Olivier Perrin, Vladimir Piterbarg, Carl-Fredrick Stein, and Bruno Torresani for useful comments. I am also very much indebted to the anonymous referees and the associate editor Christian Houdré, whose careful reading allowed great improvements in the manuscript.

\section{Appendix A}

Here we collect some technical lemma.

\section{Lemma 4.}

A function $C(s, t)$ is a stationary covariance function if and only if its Fourier transform has the form $\widehat{C}(\xi, \eta)=\delta(\xi+\eta) d \mu(\xi)$ for some finite positive measure $d \mu$.

Proof. This is a well-known result so we omit the proof.

\section{Lemma 5.}

i) Let $\sigma$ be a measure on $\mathbb{R}$. Then $\sigma \in \mathcal{S}^{\prime}(\mathbb{R})$ if and only if $\Sigma(\xi, \eta)=d \sigma(\xi) \delta(\xi+\eta) \in \mathcal{S}^{\prime}\left(\mathbb{R}^{2}\right)$.

ii) Let $\mu$ and $\nu$ be measures on $\mathbb{R}$. Then $\mu$ and $\nu$ are tempered on $\mathbb{R}$ if and only if $\mu \nabla \nu$ is tempered on $\mathbb{R}^{2}$.

Proof. Standard exercise of functional analysis.

\section{Lemma 6.}

Let $\Sigma$ be a polynomially bounded continuous function on $\mathbb{R}^{2}$ and $\rho_{j}$ be distributions in $\mathcal{S}^{\prime}(\mathbb{R})$. If

$$
\Sigma(s, t)=\sum_{j=0}^{n-1} s^{j} \rho_{j}(t)+t^{j} \overline{\rho_{j}}(s),
$$

then all the $\rho_{j}$ are continuous functions. 
Proof. If $\phi_{a}$ is an approximate identity in $\mathcal{S}^{\prime}(\mathbb{R})$ and $\psi$ a wavelet in $\mathcal{S}_{=n}(\mathbb{R})$ we have

$$
\begin{aligned}
\Sigma(0, \cdot)(\psi) & =\lim _{a \rightarrow 0} \Sigma\left(\phi_{a} \otimes \psi\right) \\
& =\lim _{a \rightarrow 0} \sum_{j=0}^{n-1} \int s^{j} \phi_{a}(s) d s \rho_{j}(\psi) \\
& =\rho_{0}(\psi) .
\end{aligned}
$$

Every function in $\mathcal{S}_{=n}(\mathbb{R})$ is the $n$th derivative of a function in $\mathcal{S}_{0}(\mathbb{R})$, which is dense in $\mathcal{S}(\mathbb{R})$. This means that $D^{n} \rho_{0}=D^{n} \Sigma(0, \cdot)$ and thus $\Sigma(0, \cdot)$ and $\rho_{0}$ differ only by a polynomial of order $n$, which implies that $\rho_{0}$ is continuous. Now suppose $\psi \in \mathcal{S}_{=n-1}(\mathbb{R})$. Then for all $b$,

$$
\begin{aligned}
\Sigma(b, \cdot)(\psi) & =\lim _{a \rightarrow 0} \Sigma\left(\phi_{b a} \otimes \psi\right) \\
& =\lim _{a \rightarrow 0} \sum_{j=0}^{n-1} \int s^{j} \phi_{b a}(s) d s \rho_{j}(\psi)+\int t^{n-1} \psi(t) d t \rho_{n-1}\left(\phi_{b a}\right) \\
& =\sum_{j=0}^{n-1} b^{j} \rho_{j}(\psi)+\int t^{n-1} \psi(t) d t \lim _{a \rightarrow 0} \rho_{n-1}\left(\phi_{b a}\right) .
\end{aligned}
$$

Now $\rho_{n-1}\left(\phi_{b a}\right)=\rho_{n-1} * \tilde{\phi}_{a}(b) \rightarrow \rho_{n-1}(b)$ in $\mathcal{S}^{\prime}(\mathbb{R})$ and thus we have the following identity in $\mathcal{S}^{\prime}(\mathbb{R})$ :

$$
\Sigma(b, \cdot)(\psi)-\sum_{j=0}^{n-1} b^{j} \rho_{j}(\psi)=\int t^{n-1} \psi(t) d t \rho_{n-1}(b) .
$$

Since the left-hand side is a continuous function of $b$, this shows that $\rho_{n-1}$ is continuous. To prove the continuity of $\rho_{n-2}$, we take a wavelet $\psi$ in $\mathcal{S}_{=n-2}(\mathbb{R})$, and proceed as above.

\section{Lemma 7.} we have

Let $\alpha>0$ and $n$ the unique integer such that $n \leq \alpha<n+1$. Then for all integer $0 \leq k \leq n$

$$
\left|\sum_{j=0}^{L} j^{k} \pi_{j}^{\alpha}\right| \leq O\left(L^{-(\alpha-k)}\right), L \rightarrow+\infty,
$$

where the $\pi_{j}^{\alpha}$ are the generalized Binomial coefficients defined in (1.4).

First note that $\sum_{j=0}^{\infty} \pi_{j}^{\alpha}=0$. Since $\left|\pi_{j}^{\alpha}\right| \sim j^{-\alpha-1}$, it follows that

$$
\left|\sum_{j=0}^{L} \pi_{j}^{\alpha}\right|=\left|\sum_{j=L+1}^{\infty} \pi_{j}^{\alpha}\right| \leq O\left(L^{-\alpha}\right), L \rightarrow+\infty .
$$

Now using the recurrence relation $\Gamma(x+1)=x \Gamma(x)$ for the Gamma function and the definition (1.4) of the binomial coefficients, a straightforward computation gives

$$
\sum_{j=0}^{L} j(j-1) \ldots(j-k+1) \pi_{j}^{\alpha}=\frac{\Gamma(k-\alpha)}{\Gamma(-\alpha)} \sum_{j=0}^{L-k} \pi_{j}^{\alpha-k},
$$

for all $0 \leq k \leq n$. Applying (7.2) to $\alpha-k$, it follows that

$$
\left|\sum_{j=0}^{L} j(j-1) \ldots(j-k+1) \pi_{j}^{\alpha}\right| \leq O\left(L^{-(\alpha-k)}\right), L \rightarrow+\infty,
$$

for all $0 \leq k \leq n$. Now we can write $j^{k}=j(j-1) \ldots(j-k+1)+P_{k-1}(j)$, where $P_{k-1}$ is a polynomial of degree at most $k-1$. By induction starting from $k=2$, this proves (7.2). 


\section{Lemma 8.}

Let $\alpha>0$ and $n$ the unique integer such that $n \leq \alpha<n+1$. Let the function $g$ be at least $n+1$ times continuously differentiable and, together with its $n+1$ first derivatives, rapidly decreasing at infinity. Then for the function

$$
\rho_{\tau}(t)=\tau^{-\alpha} \sum_{j=0}^{\infty} \pi_{j}^{\alpha} g(t-\tau j),
$$

we have the uniform estimation

$$
\sup _{\tau>0}\left|\rho_{\tau}(t)\right| \leq O\left(\frac{1}{1+|t|^{\alpha+1-\epsilon}}\right)
$$

for $\epsilon$ arbitrarily small. For the function

$$
\rho_{\tau}^{a b s}(t)=\tau^{-\alpha} \sum_{j=0}^{\infty}\left|\pi_{j}^{\alpha}\right| g(t-\tau j) \mid
$$

we have only

$$
\left|\rho_{\tau}^{a b s}(t)\right| \leq C_{\tau, \epsilon} \frac{1}{1+|t|^{\alpha+1-\epsilon}}
$$

for some constant $C_{\tau, \epsilon}$ depending on $\tau$ and $\epsilon$.

Proof. Let $\epsilon>0$. First consider $t \geq 0$. The above sum can be split into three terms:

$$
\begin{aligned}
\rho_{\tau}(t) & =\tau^{-\alpha}\left\{\sum_{\tau j<t-t^{\epsilon}}+\sum_{|t-\tau j| \leq t^{\epsilon}}+\sum_{\tau j>t+t^{\epsilon}}\right\} \pi_{j}^{\alpha} g(t-\tau j) \\
& =A_{1}+A_{2}+A_{3} .
\end{aligned}
$$

Let us estimate each term separately. By Taylor's formula with Lagrange remainder at order $n$ we have

$$
g(t-\tau j)=\sum_{k=0}^{n} \frac{(-\tau j)^{k}}{k !} g^{(k)}(t)+\frac{(-\tau j)^{n+1}}{(n+1) !} g^{(n+1)}\left(t_{\tau j}\right),
$$

where $t_{\tau j}$ is some point in the interval $[t-\tau j, t]$ and $g^{(k)}$ stands for the $k$ th derivative of the function g. Hence,

$$
\begin{aligned}
A_{1} & =\tau^{-\alpha} \sum_{k=0}^{n} g^{(k)}(t) \frac{(-\tau)^{k}}{k !} \sum_{\tau j<t-t^{\epsilon}} j^{k} \pi_{j}^{\alpha}+\frac{(-1)^{n+1}}{(n+1) !} \tau^{n+1-\alpha} \sum_{\tau j<t-t^{\epsilon}} j^{n+1} \pi_{j}^{\alpha} g^{(n+1)}\left(t_{\tau j}\right) \\
& =A_{1}^{\prime}+A_{1}^{\prime \prime}
\end{aligned}
$$

In view of Lemma 7 we have $\left|\sum_{\tau j<t-t^{\epsilon}} j^{k} \pi_{j}^{\alpha}\right| \leq O(t / \tau)^{k-\alpha}$ for all $0 \leq k \leq n$. Using the rapid decay of $g^{(k)}$, this implies

$$
\left|A_{1}^{\prime}\right| \leq O(1) \frac{1}{1+t^{\alpha+1}} .
$$

To estimate the term $A_{1}^{\prime \prime}$, note that $t_{\tau j}$ lies in the interval $\left[t^{\epsilon}, t\right]$ whenever $\tau j<t-t^{\epsilon}$. Since $g^{(n+1)}$ is rapidly decreasing, we have the uniform estimation $\left|g^{(n+1)}\left(t_{\tau j}\right)\right| \leq O\left(\frac{1}{1+t^{l \epsilon}}\right) \leq O\left(\frac{1}{1+t^{2+n}}\right)$ for 
some $l$ large enough. Using $\left|\pi_{j}^{\alpha}\right| \sim j^{-\alpha-1}$ it comes

$$
\begin{aligned}
\left|A_{1}^{\prime \prime}\right| & \leq O\left(\tau^{n+1-\alpha}\right) \frac{1}{1+t^{2+n}} \sum_{j \leq t / \tau} j^{n-\alpha} \\
& \leq O\left(\tau^{n+1-\alpha}\right) \frac{1}{1+t^{2+n}}\left(\frac{t}{\tau}\right)^{n+1-\alpha} \\
& \leq O(1) \frac{1}{1+t^{\alpha+1}} .
\end{aligned}
$$

For the second term $A_{2}$ we use simply the boundedness of $g$ :

$$
\begin{aligned}
\left|A_{2}\right| & \leq O\left(\tau^{-\alpha}\right) \sum_{|t-\tau j| \leq t^{\epsilon}} j^{-\alpha-1} \\
& \leq O\left(\tau^{-\alpha}\right)\left(\frac{t}{\tau}\right)^{-\alpha-1} \operatorname{card}\left\{j: \sum_{|t-\tau j| \leq t^{\epsilon}}\right\} \\
& \leq O(\tau) t^{-\alpha-1} \tau^{-1} t^{\epsilon} \\
& \leq O(1) \frac{1}{1+t^{\alpha+1-\epsilon}} .
\end{aligned}
$$

For the last term we use again the rapid decay of $g$ together with the asymptotic form of $\left|\pi_{j}^{\alpha}\right|$.

$$
\begin{aligned}
\left|A_{3}\right| & \leq \tau^{-\alpha} \sum_{\tau j>t+t^{\epsilon}} j^{-\alpha-1}|g(t-\tau j)| \\
& \leq O\left(\tau^{-\alpha}\right) \frac{1}{\left(1+t^{\epsilon}\right)^{l}} \sum_{\tau j>t+t^{\epsilon}} j^{-\alpha-1} \\
& \leq O\left(\tau^{-\alpha}\right) \frac{1}{1+t} \sum_{\tau j>t+t^{\epsilon}} j^{-\alpha-1} \\
& \leq O\left(\tau^{-\alpha}\right) \frac{1}{1+t}\left(\frac{1+t}{\tau}\right)^{-\alpha} \\
& \leq O(1) \frac{1}{1+t^{\alpha+1}} .
\end{aligned}
$$

For $t<0$, the method is analogous but one has to consider the cases $\tau j<|t|^{\epsilon}$ and $\tau j \geq|t|^{\epsilon}$ instead. The proof of (7.5) is in the same spirit and we omit it.

\section{Appendix B}

Here we recall some properties of homogeneous distributions. For any real $\alpha$, let

$$
\kappa_{+}^{\alpha}(x)=\left\{\begin{array}{l}
x^{\alpha} \text { if } x>0, \\
0 \text { else }
\end{array}, \kappa_{-}^{\alpha}(x)=\left\{\begin{array}{l}
|x|^{\alpha} \text { if } x<0, \\
0 \text { else }
\end{array} .\right.\right.
$$

Clearly, for $\alpha>-1, \kappa_{ \pm}^{\alpha}$ define tempered distributions. Whenever $\alpha<-1$ is not a negative integer, $\kappa_{ \pm}^{\alpha}$ can still be extended to distributions in $\mathcal{S}^{\prime}(\mathbb{R})$ (a complete study of these functions can be found in [14]) by setting

$$
\kappa_{ \pm}^{\alpha}(\varphi)=\int_{0}^{+\infty} x^{\alpha}\left[\varphi( \pm x)-\varphi(0)- \pm x \varphi^{\prime}(0)-\ldots-( \pm 1)^{n-1} \frac{x^{(n-1)}}{(n-1) !} \varphi^{(n-1)}(0)\right] d x
$$


where $-n-1<\mathfrak{R} \alpha<-n$. Their Fourier transform is given by

$$
\widehat{\kappa_{ \pm}^{\alpha}}=i \Gamma(\alpha+1)\left(e^{i \alpha \pi / 2} \kappa_{ \pm}^{-\alpha-1}-e^{-i \alpha \pi / 2} \kappa_{\mp}^{-\alpha-1}\right)
$$

In particular, the Fourier transform of the function $|x|^{\alpha}:=\kappa_{+}^{\alpha}(x)+\kappa_{-}^{\alpha}(x)$ is $C_{\alpha}|\xi|^{-\alpha-1}$, with $C_{\alpha}=-2 \Gamma(\alpha+1) \sin (\alpha \pi / 2)$.

\section{References}

[1] Abry, P., Flandrin, P., Taqqu, M.S., and Veitch, D. (1999). Wavelets for the analysis, estimation and synthesis of scaling data, In Self-Similar Network Traffic and Performance Evaluation, to appear.

[2] Averkamp, R. and Houdré, C. A note on the discrete wavelet transform of second-order processes, IEEE Trans. Inform. Theory, to appear.

[3] Averkamp, R. and Houdré, C. (1998). Some distributional properties of the continuous wavelet transform of random processes, IEEE Trans. Inform. Theory, 44(3), 1111-1124.

[4] Brockwell, P.J. and Davis, R.A. (1987). Time Series: Theory and Methods, Springer-Verlag, New York.

[5] Cambanis, S. and Houdré, C. (1995). On the continuous wavelet transform of second-order random processes, IEEE Trans. Inform. Theory, 41(3), 628-642.

[6] Cheng, B. and Tong, H. (1996). A Theory of Wavelet Representation and Decomposition for a General Stochastic Process, Number 115 in Lectures Notes in Statistics. Springer-Verlag, New York, 115-129.

[7] Dijkerman, R.W. and Mazumdar, R.R. (1994). On the correlation structure of the wavelet coefficients of fractional brownian motion, IEEE Trans. Inform. Theory, 40(5), 1609-1616.

[8] Dijkerman, R.W. and Mazumdar, R.R. (1994). Wavelet representations of stochastic processes and multiresolution stochastic models, IEEE Trans. Signal Proc., 42(7), 1640-1652.

[9] Doob, J.L. (1953). Stochastic Processes, Wiley Publications in Statistics, Wiley, New York.

[10] Flandrin, P. (1989). On the spectrum of fractional brownian motion, IEEE Trans. Inform. Theory, 35(1), 197-199.

[11] Flandrin, P. (1992). Wavelet analysis and synthesis of fractional brownian motion, IEEE Trans. Inform. Theory, 38(2), 910-917.

[12] Folland, G.B. (1984). Real Analysis, John Wiley \& Sons, New York.

[13] Gel'fand, I.M. (1955). Generalized random processes, Dokl. Akad. Nauk. SSSR, 100, 853.

[14] Gel'fand, I.M. and Shilov, G.E. (1964). Generalized Functions. Vol. 1. Academic Press [Harcourt Brace Jovanovich Publishers], New York, [1977]. Properties and operations, Translated from the Russian by Eugene Saletan.

[15] Gel'fand, I.M. and Vilenkin, N.Ya. (1964). Generalized Functions. Vol. 4. Academic Press [Harcourt Brace Jovanovich Publishers], New York, [1977]. Applications of harmonic analysis, Translated from the Russian by Amiel Feinstein.

[16] Granger, C.W.J. and Joyeux, R. (1980). An introduction to long-memory time series models and fractional differencing, J. Time Ser. Anal., 1(1), 15-29.

[17] Hosking, J.R.M. (1981). Fractional differencing, Biometrika, 68(1), 165-176.

[18] Houdré, C. (1990). Harmonizability, v-boundedness, (2,p)-boundedness of stochastic processes, Probab. Th. Rel. Fields, 87, 167-188.

[19] Houdré, C. (1993). Wavelets, probability and statistics: some bridges, Wavelets: Mathematics and Applications, Benedetto, J. and Frazier, M., Eds., CRC Press, Boca Raton, FL, 361-394.

[20] Krim, H. and Pesquet, J.C. (1995). Multiresolution analysis of a class of nonstationary processes, IEEE Trans. Inform. Theory, 41, 1010-1020.

[21] Loève, M. (1978). Probability Theory. Il. 4th ed., Springer-Verlag, New York, Graduate Texts in Mathematics, Vol. 46.

[22] Mallat, S., Papanicolaou, G., and Zhang, Z. (1998). Adaptive covariance estimation of locally stationary processes, Ann. Statist., 26(1), 1-47.

[23] Mandelbrot, B.B. and Van Ness, J.W. (1968). Fractional Brownian motions, fractional noises and applications, SIAM Rev., 10, 422-437.

[24] Masry, E. (1993). The wavelet transform to stochastic processes with stationary increments and its application to fractional brownian motion, IEEE Trans. Inform. Theory, 39(1), 260-264.

[25] Masry, E. (1996). Convergence properties of wavelet series expansions of fractional brownian motion, Appl. Comp. Harm. Anal., 3, 239-253. 
[26] Michálek, J. (1986). Ergodic properties of locally stationary processes, Kybernetika (Prague), 22(4), 320-328.

[27] Michálek, J. (1986). Spectral decomposition of locally stationary random processes, Kybernetika (Prague), 22(3), 244-255.

[28] Michálek, J. (1989). Linear transformations of locally stationary processes, Apl. Mat., 34(1), 57-66.

[29] Pesquet-Popescu, B. (1998). Modélisation bidimensionnelle de processus non-stationnaires et application à l'étude du fond sous-marin. Ph.D. thesis, Ecole Normale Supérieure de Cachan, July.

[30] Pesquet-Popescu, B. (1999). Wavelet packet analysis of $2 d$ processes with stationary fractional increments, IEEE Trans. Inform. Theory, 1033-1039.

[31] Pesquet-Popescu, B. and Larzabal, P. (1997). 2d-self-similar processes with stationary fractional increments. In Fractals in Engineering, Tricot Levy Véhel, Lutton, Ed., Springer-Verlag, Berlin.

[32] Picinbono, B. (1974). Properties and applications of stochastic processes with stationary $n$ th-order increments, Adv. Appl. Prob., 6, 512-523.

[33] Pinsker, M.S. (1955). The theory of curves in hilbert space with stationary $n$th increments, (Russian), Izv. Akad. Nauk SSSR. Ser. Mat., 19, 319-344.

[34] Pinsker, M.S. and Yaglom, A.M. (1954). Random processes with stationary increments of the $n$th order, Dokl. Akad. Nauk. SSSR, 94, 385-388.

[35] Ramanathan, J. and Zeitouni, O. (1991). On the wavelet transform of fractional brownian motion, IEEE on Information Theory, 37(4), 1156-1158.

[36] Rozanov, Yu.A. (1959). Spectral analysis of abstract functions, Theor. Probab. Appl.

[37] Schwartz, L. (1950). Théorie des Distributions, Hermann, Paris.

[38] Silverman, R.A. (1957). Locally Stationary Random Processes. Div. Electromag. Res., Inst. Math. Sci., New York University. Res. Rep. No. MME-2.

[39] Tewfik, A.H. and Kim, M. (1992). Correlation structure of the discrete wavelet coefficients of fractional brownian motion, IEEE Trans. Inform. Theory, 38(2), 904-909.

[40] Veitch, D. and Abry, P. (1998). A wavelet based joint estimator for the parameters of Ird., IEEE Trans. Info. Th., special issue "Multiscale Statistical Signal Analysis and its Application."

[41] Winkler, H. (1993). Integral representation for stochastic processes with $n$th stationary increments, Math. Nachr, 163, 35-44.

[42] Wong, P.W. (1993). Wavelet decomposition of harmonizable random processes, IEEE Trans. Inform. Theory, 39(1), 7-18.

[43] Yaglom, A.M. (1958). Correlation theory of processes with random $n$th increments, Am. Math. Soc. Transl., 8(2), 87-141.

[44] Yaglom, A.M. (1987). Correlation Theory of Stationary and Related Random Functions, Vol. 1, Springer-Verlag, New York.

[45] Yaglom, A.M. and Pinsker, M.S. (1953). Random processes with stationary increments of the $n$th order, Dokl. Akad. Nauk. SSSR, 90, 731-734.

Department of Mathematics and Statistics, Chalmers University of Technology, S-41296 Gothenburg, Sweden

Laboratoire d’Optique Electromagnétique. Faculté des Sciences de Saint-Jérôme, case 162. F-13397 Marseille cedex 20 e-mail: caguerin@loe.u-3mrs.fr 